\title{
Elastic Inflatable Actuators for Soft Robotic Applications
}

Benjamin Gorissen ${ }^{a}$, Dominiek Reynaerts ${ }^{a}$, Satoshi Konishi ${ }^{b}$, Kazuhiro Yoshida ${ }^{c}$, Joon-wan Kim, Michael De Volder ${ }^{\mathrm{a}, \mathrm{d}}$

${ }^{a}$ Department of Mechanical Engineering, Katholieke Universiteit Leuven \& Flanders Make, Celestijnenlaan $300 B, 3001$ Leuven, Belgium

${ }^{b}$ Department of Mechanical Engineering, Ritsumeikan University, 1-1-1 Noji-higash, Kusatsu, Shiga 525-8577, Japan

c Yoshida \& Kim Laboratory, Tokyo Institute of Technology, 4259 Nagatsuta-cho, Midori-ku, Yokohama 226-8503, Japan

${ }^{d}$ Institute for Manufacturing, Dept. of Engineering, University of Cambridge, 17 Charles Babbage Road, Cambridge, CB3 OFS, UK

ABSTRACT

The $20^{\text {th }}$ century's robotic systems have been made out of stiff materials and much of the developments in the field have pursued ever more accurate and dynamic robots which thrive in industrial automation settings and will probably continue to do so for many decades to come. However, the $21^{\text {st }}$ century's robotic legacy may very well become that of soft robots. This emerging domain is characterized by continuous soft structures that simultaneously fulfil the role of robotic link and robotic actuator, where prime focus is on design and fabrication of the robotic hardware instead of software control to achieve a desired operation. These robots are anticipated to take a prominent role in delicate tasks where classic robots fail, such as in minimally invasive surgery, active prosthetics and automation tasks involving delicate irregular objects. Central to the development of these robots is the fabrication of soft actuators to generate movement. This paper reviews a particularly attractive type of soft actuators that are driven by pressurized fluids. These actuators have recently gained substantial traction on the one hand due to the technology push from better simulation tools and new manufacturing technologies including softlithography and additive manufacturing, and on the other hand by a market pull from the applications listed above. This paper provides an overview of the different advanced soft actuator configurations, their design, fabrication and applications.

\section{Introduction}

It is anticipated that future robotic systems will carry out increasingly complex tasks and will interact more closely with humans. As a result, advanced robots that are inherently safe have been intensively investigated by the robotics community. Safety features can be programmed in the software of classic rigid robots to impose force limits and artificial compliance. While impressive advances have been achieved using this approach [1], designing robots that are made out of soft materials, such as rubber instead of metals, offer a new paradigm to make man-machine interaction inherently safe and to mimic soft biologic systems [2]. Evolving from hard to soft robots also requires an evolution from software intelligence to hardware intelligence, where functionalities are 'programmed' in the overall hardware design. In particular, the actuators used to move soft-robots typically embed information on the motion path, that enable the robot to inherently adapt to the objects it is interacting with, as well as to operate safely in its environment. 
This paper will review a specific type of soft robotic actuators powered by a pressurized fluid (gas or liquid). These are referred to as elastic inflatable actuators (EIAs). These advanced actuators have the advantage of achieving large strokes, very little friction and exhibit distributed force generation due to Pascal's principle [3]. Further, fluidic actuation is not relying on any high electric or magnetic fields (unlike electrostatic, piezoelectric actuators, electroactive polymers and electromagnetic motors) or high-temperatures (unlike shape memory alloys and thermal actuators) which can be detrimental for robotic orthotics or robotic surgery tools [4]. For the latter applications, EIAs can be pressurized using biocompatible fluids without altering the actuation principle. A more general overview on soft robotics is available in references $[2,5]$.

With earliest reports on EIAs dating back to 1919 [6], various actuator designs have emerged which can roughly be classified according to their motion path [7]: contracting actuators, expanding actuators, twisting actuators and bending actuators. All these actuators rely on the same underlying physical principles, which consist of a carefully engineered anisotropic flexible structure that is inflated by a pressurized fluid. Depending on the anisotropy that is programmed in the actuator structure, different motions are created [810], as can be seen in Fig. 1. Expanding (Fig.1 a-d) and contracting actuators (Fig.1 e-h) have a structurally symmetrical cross-section, where the former typically rely on the pressurized expansion of the flexible structure to generate a linear motion. A contracting motion is usually achieved by strain-limiting elements (fibres) that transform the inflation into a lengthwise contraction (e.g. McKibben actuators [11]). Twisting deformations (Fig.1 i-l) are created by point symmetrical boundary conditions, which can be imposed by spiralling fibres $[12,13]$. Bending actuation (Fig. $1 \mathrm{~m}-\mathrm{p}$ ) is achieved by designing an asymmetric actuator cross section, where bending is directed towards the actuator section with the highest bending stiffness $[14,15]$. Since these actuators only differ in boundary conditions, analogue production processes have been developed for the four types of actuators discussed above. Combining these designs makes it possible to achieve more complex motions using only one actuator [16]. This often results in de-localized or continuous actuators and morphing of the robot frame and the actuators into a complex multi-functional and integrated body with new functionalities. An example of this approach can be seen in Fig.1d [17], where multiple expanding actuators are circularly arranged to form a continuous rotary fluidic actuator, resembling an electric stepper motor.

The materials used for large stroke elastic inflatable actuators are characterized by large strains and low elastic moduli. Typical elastic moduli are in the range of $10^{5}-10^{7} \mathrm{~Pa}$ for deformable materials (usually silicones) and $10^{8}-10^{10} \mathrm{~Pa}$ for strain limiting layers [2]. Besides extremely large attainable hyperelastic strains for silicones of up to $1000 \%$ (Ecoflex ${ }^{\circledR} 5$ ), material properties are found to be dependent on strain rate and vary between batches. Further, there is an initial straining effect and stress relaxation behaviour is reported in literature [18]. However, after the initial straining effect, material properties are practically unaffected by cyclic loading with reports of actuator performance remaining stable over more than a 1 million cycles [19]. It should be noted that these cyclic test have only been 
conducted at moderate straining levels, and high strain cyclic behaviour requires further investigation. Further, the frequency at which these cycling tests can be performed is limited by the actuation fluid that needs to pass through relatively narrow tubing, resulting in maximal reported full-stroke bandwidths in the order of $10 \mathrm{~Hz}[19,20]$, or $20 \mathrm{~Hz}$ when tubing is eliminated [21].

In what follows, this paper will detail four aspects of EIAs: design and modelling, fabrication techniques, control, and applications. The focus will be on advanced EIAs with a bending deformation because these are by far the most intensively investigated and similar techniques are reported for other types of EIAs.

\section{Design and modelling}

Bending EIAs achieve their deformation via a flexible asymmetrical axial cross section surrounding an inflatable void. This asymmetry causes the structure to bend when inflated, as illustrated in Fig. 2. This figure also illustrates three types of axial asymmetry that have been developed in literature for bending EIAs: multi-material, corrugated membranes and eccentric void asymmetries. However, it is common practice to combine multiple types of asymmetry in a single actuator design. Multi-material asymmetry is achieved by combining materials with different Young's moduli that are asymmetrically organized around a central void. In practice this is done by either altering compositions of the rubber [14] or by embedding stiff strain limiting materials such as fibres [8, 22] or paper [23] in the actuator. Asymmetry by corrugated membranes is achieved by incorporating folds on one side of the actuator cavity which expand more readily under pressure [19, 24-26]. Eccentric void asymmetry is obtained when the pressure chamber is placed eccentrically in the surrounding elastic structure $[27,28]$.

The ability to design and model soft actuators is particularly important because in soft robotics the motion path is typically programmed in the design of the actuator. Empirical rules of thumb, as well as more advanced analytical models and finite element models (FEM) have been developed as discussed below [29].

\subsection{Empirical methods}

Empirical guidelines have been reported in literature to design EIAs which allow for fast actuator designs. In a planar eccentric configurations, an optimal force generation is reported when the ratio of length to width of the inflatable void is around 10 [30], while the bending stroke is optimized if the bending rigidity between layers differs with a factor of 2 to 3 [15]. Further, it is reported that bending stroke increases when divided balloons are used [31]. Finally, some unexpected actuation properties have been reported for different types of asymmetry [32]. Publications on eccentric void actuators describe a bidirectional stroke, where at low pressure the deformation is directed towards the low stiffness side and at high pressure towards the other side $[15,28,33]$. This effect can be avoided by using multimaterial asymmetry $[14,28]$ and should not be confused with the negative stroke that bending ElAs exhibit when exposed to vacuum [34,35]. Drawbacks of eccentric actuators are 
their large radial expansion [36] and a slow actuation, which can be improved by using corrugated membranes [19].

\subsection{Analytical methods}

Euler-Bernoulli's beam theory is by far the most widespread model used in literature to describe the overall static deformation of bending actuators. In this theory, the bending actuator is modelled as an ideal beam loaded with a pure moment at its edges, originating from the axial component of the loading pressure and counteracted by the bending stiffness (EI) of the beam (with $E$ the Young's modulus and I the second moment of area). Although Euler-Bernoulli's beam theory is not applicable for large bending deformations, where crosssectional planes do not stay perpendicular to the actuator axis, it has been showed in literature that it is a useful tool for basic qualitative analysis of bending EIAs (multi-material asymmetry [37], eccentric void asymmetry [38], three-chambered actuators [39]). A particular challenge in these simulations is that the actuator's cross section changes with inflation, and therefore, the unpressurized cross section only provides a good approximation at low pressures $[39,40]$. Improvements can be made by updating the actuator's crosssection as it inflates [41-43]. Finally, several external loads on the actuators have been modelled in literature (e.g. gravitational loading [38], tip loading [44-46], edge loading [47] and dynamic effects $[38,48])$.

In the case of more intricate cross-sectional changes (e.g. corrugated membranes), it was found experimentally that the relation between curvature and bending moment becomes very complex [44], indicating that Euler-Bernoulli's beams theory is not valid anymore. Further, all previous models assume a fixed Young's modulus, which is often a major source of error since most soft materials show a complex stress-strain behaviour.

\subsection{Finite element method}

To cope with the limitations above more accurate finite element method (FEM) models have been developed [12], which allow for optimising actuator geometry [27, 49, 50]. Different FEM implementations have been suggested in literature as summarized in Table 1. In general, nonlinear FEM solvers are preferred as they can cope with large deformations and material nonlinearities. A key parameter is the used hyperelastic material model for describing the rubber-like material, because low order material models can only accurately describe material properties at low strains $[19,31]$. The accuracy of the model is typically improved when material parameters are first experimentally measured [51] either uniaxially $[19,24,35,52,53]$ or biaxially [50]. Further, few dynamic FEM models are reported in literature $[52,54]$, and in general complex soft robots topologies remain challenging to model. Lastly, the concept of evolutionary optimisation algorithms has also been implemented to shape actuators. In this approach, FEM models are used to determine the "fitness" of different designs [29], which are then combined to create sometimes unintuitive designs with improved performances [55]. 


\section{Fabrication}

The performance of EIAs is closely linked to the manufacturing techniques used to fabricate the actuators as these need to be able to process elastic materials in leak-tight complex shapes. As a result, advances in the field of soft robotics have been directly linked to the development of new manufacturing technologies. For instance, (i) The advent of soft lithography and its ability to shape rubber structures at the micrometre scale has been transformative for the development of EIAs, in particular for applications requiring microscale robots such as for minimally invasive surgery. (ii) Additive manufacturing provided a second thrust for increasingly complex soft robot designs. Fig. 3 provides a more detailed account of how the design of soft actuators evolved with the availability of new manufacturing processes. While we do not know the fabrication process of initial EIAs patented as early as 1967 [56], Suzumori et al. reported a three-chambered 3 degree of freedom (DOF) bending actuator by moulding in 1989 [57, 58]. These actuators were reduced by a tenfold in size ( $1 \mathrm{~mm}$ diameter) using the same process by 1991 [39]. The first EIAs fabricated by stereolithographic additive manufacturing were reported as early as in 1994 [59]. In 1997, an extrusion-compatible design was proposed which allows for scale-up fabrication of these advanced actuators [27]. Hirai et al. and Ikuta et al. developed new asymmetric designs by locally placing extension limiting fibres in 2000 [8], and by local corrugated membranes in 2002 [60]. Kawai et al. introduced a planar bending EIA design, using MEMS processes in 2000 [61], which was further developed by Konishi et al. in 2005 using a soft lithography process [62], giving way to a much smaller planar actuator design. Other means of producing planar moulds, micromachining and 3D printing, have been reported by Ogura et al. in 2009 [35] and Ilievski et al. in 2011 [63]. The smallest bending EIAs in literature (diameter $0.4 \mathrm{~mm}$ ) were produced by Ikuta et al. using a micro-embossing process in 2002. Production processes which eliminate the need for sealing the inflatable void have been reported by Lu et al. in 2003 using a full lithography process [64] and by Katzschmann et al. in 2014 using a lost wax production technique. This is important since the sealing or bonding of the two layers often results in leakage and failure of the actuator.

In what follows we describe in more detail four production processes that are representative for the fabrication of most ElAs. As shown in Fig. 4 A, each production processes occupies its own design space in terms of dimensions, geometry, and the method for generating asymmetry (Fig. 2) as will be discussed in more detail below. Overall, these asymmetry generating methods differ in topological complexity, which has an effect on size limitations.

\subsection{D moulding (Fig. 4 B)}

In 3D moulding processes, a single mould defines all actuator sidewalls, and an inflatable volume is created by sealing the actuator with a cap that is typically on the opposite side of the pressure supply (see Fig. 4 B). This process enables large aspect ratio actuators (> 50 in some cases [43]) where the demoulding step is often the limiting factor. Corrugated membranes and other re-entrant structures are not possible with this approach unless 
complex retractable moulds are used. Alternatively, asymmetry can be introduced by adhering strain-limiting fibres to axisymmetric rubber tubes [8, 9, 22, 65-67] or by placing asymmetric Nitinol structures in the inflatable void [68]. Most moulds are fabricated either by micromachining or additive manufacturing:

- Micromachined moulds were first developed by Suzumori et al., who used an electrical discharge machining process to fabricate two-part moulds for radial fibre reinforced actuators with diameters down to $1 \mathrm{~mm}[12,39,45,48,50,52,57,69]$. Later alterations of the process removed the need for a sealing cap [41] and improved the de-moulding process [70]. Finally, Paek et al. [43] developed a simplified dip-coating process and Schwörer et al. [71] reported a blow moulding process.

- Additive manufacturing processes have been used to fabricate moulds for complex 3D actuators [72] as well as full robotic systems consisting of multiple bending actuator segments [73]. Due to the typical surface roughness of additive manufacturing, the outer mould is usually composed of different parts that can be disassembled [74, 75]. To eliminate bonding of different actuator components, Marchese et al. [76, 77] developed a lost-wax casting process where the inflatable void is shaped using a sacrificial wax core. Also multimaterial vertical moulded actuators made by sequentially casting different rubbers have been reported [73]. To date, actuators that are fabricated by additive manufacturing have typical outer dimensions of $10 \mathrm{~mm}$ and up.

\subsection{D moulding (Fig. 4 C)}

While 3D moulds allow for complex actuator designs, they are sometimes limited by the de-moulding process and are typically used for millimetre scale or larger actuators. Alternatively, fabricating actuators by joining 2D planar moulded pieces allows to create smaller actuators with different types of asymmetry, for instance by joining two layers with different mechanical properties, as is shown in the inset of figure of Fig. 4 C. Key to this process is the ability to create strong, leak-free bonds between actuator layers. Typically, one of the bonded layers has a void geometry defined inside, while the other layer is flat to avoid the need of bond alignment. Following techniques are commonly used to fabricate the 2D moulds:

- Additive manufacturing is used for relatively large moulds [78] and can be used to create corrugated actuators $[19,79]$ as well as designs combining eccentric void and material asymmetry $[53,63,80]$. The asymmetry of these actuators was further engineered by incorporating polyaramid fibres [81, 82], paper [23], textile [83-85], ABS plastic [86, 87] and a woven sheet of PET [88] in the design.

- Soft-lithography is particularly suitable for miniaturising 2D moulding [89]. Konishi et al. developed actuators by spin coating PDMS over a lithographically patterned SU8 mould [62]. The PDMS layer is cured and then peeled from the mould and bonded to a flat spin coated PDMS sheet. The two layers are bonded with the same processes as used in microfluidic PDMS devices (e.g. using partially cured PDMS [90, 91], or plasma and UV-O activation $[24,28])$. The smallest reported features for these actuators are in the order of a 
few 10's of micrometres and are currently limited by the manual transfer and bonding steps. Further, methods are reported to control the thicknesses of the actuator more precisely using spacers instead of spin-coating $[92,93]$.

- Micromachining (eg. 3 or 5 axis milling) has been reported for making simple 2D moulds [15, 94] and complex corrugated geometries [24, 35, 95]. A related planar microembossing process (MeME-X) was introduced by lkeuchi et al. [25, 96], where a master mould is pressed against a thermoplastic membrane at the glass transition temperature.

- Laser cutting can also be used to make planar moulds [97, 98]. Tolley et al. [99] fabricated $65 \mathrm{~cm}$ long actuators with moulds made by waterjet and laser-cutting.

\subsection{Full lithography}

To further decrease dimensions, fully lithographic production processes have been developed that exclude manual production steps. Gorissen et al. [100] used a sacrificial etch layer shaped as the inflatable void in between two layers of PDMS, while the outside geometry of the actuator is cut out using a reactive ion etching process, allowing structures with planar dimensions of a few micrometres to be produced. Alternatively, Lu et al. [64] used a conformal parylene coating to form inflatable balloons between overhanging silicon beams.

\subsection{Additive manufacturing (Fig. 4 D)}

While all production processes discussed above are essentially 2.5D processes, additive manufacturing makes it possible to fabricate 3D EIAs. In contrast to the 3D printed moulds discussed earlier, the actuators in this case are directly printed. In literature, three additive manufacturing methods are reported that allow printing of flexible structures. Stereolithography is used to produce $\mathrm{mm}$ - to $\mathrm{cm}$-sized bending EIAs [26, 59, 101]. For bigger $(>10 \mathrm{~mm})$ actuators, Polyjet printing was reported, which has the advantage that multiple materials can be printed, for instance to make expansions limiting hoops ( $\sim 3.5 \mathrm{MPa}$ ) within the actuator $(E \sim 0.45 \mathrm{MPa})[38,102,103]$ or to realize unibody fingers with rigid bones and flexible joints in one process [104]. Other additive manufacturing techniques, selective laser sintering and fused deposition modelling (FDM), have also been reported for fabricating bending EIAs [105, 106]. Additionally, rigid structures can be adhered to inflatable volumes to direct the deformation in a so-called pouch motor design $[107,108]$. Further, the internal void of the actuator can directly be formed out of inflatable porous foams $[29,109,110]$. In another additive manufacturing inspired process, inflatable voids out of fugitive ink are directly printed (EMB3D printing) inside a still liquid polymer material [111]. After the polymer material hardens, the ink is removed via auto-evacuation leaving inflatable voids and channels behind.

In order to evaluate different actuator configurations and fabrication methods, an objective metric is needed that can be computed with data found in literature. Force density is an obvious metric $[28,112]$, however most publications report deformations rather than 
forces. Strokes are difficult to compare because they depend on the actuator size and pressure applied and therefore we propose a bending coefficient to compare different EIAs. According to simplified Euler-Bernoulli beam theory, there is a linear relation between input pressure and curvature. The ratio of curvature over input pressure can be seen as a mechanical transmission coefficient that links input pressure to output curvature and is independent of the actuator size. For real bending EIAs, this relation is not linear over pressure, however different actuators can be compared to each other when this ratio is computed at a certain bending deformation, taken to be $90^{\circ}$ in this paper. The results of this literature survey is plotted in Fig. 5, where bending coefficient is plotted versus the actuator aspect ratio (length of the actuator over its width). In Fig. 5A, actuators are compared according to production process while in Fig. 5B actuator designs are compared. These figures indicate that despite lots of effort put in corrugated membrane and multi-material actuators (using an inextensible layer or using different elastomers), they are almost all outperformed in terms of bending coefficient by actuators that only use an eccentric void topology. However, as previously mentioned, corrugated actuators respond faster [19]. Further, 2D moulded actuators tend have a higher bending coefficient than other production processes, this is partly due to for instance limitations in materials available for additive manufacturing.

\section{Control}

Initially, the soft robotics community tried to apply known control strategies to soft actuators. Feedforward control schemes were implemented where model identification was done using the analytical models described above [27], using neural network regression [78] and using polynomial based methods $[113,114]$. Typically, these approaches achieved accuracies of a few millimetres at best. Alternatively, feedback control schemes enabling error compensation were also pursued in literature: P-control [12, 45, 48]; PI-control for point-to-point movement and path tracking experiments [86], as well as in whole arm planning experiments in 2D [74] and 3D [75]; PID-control [115] and even more advanced controllers using dynamic system identification $[116,117]$ in conjunction with LQR and LQG control $[117,118]$. Essential for these feedback control schemes is the availability of sensory feedback of the actuator's shape. This can be achieved using external measurement devices, e.g. a system of cameras and localized markers $[12,74,75,86]$, a cable measurement system [119] and electromagnetic tracking $[114,116]$ or by using sensors that are integrated within the EIAs [120]. However, internal systems do not provide direct feedback of the end-effector position but for instance register strain inside the actuators which is often correlated to actuator deformation or end-effector position. Examples include piezoelectric sensors [102], [121], piezoresistive sensors [98, 115], and fluidic sensors [122]. Because of the absence of coulomb friction, EIAs can achieve sub-micrometer positioning accuracies using feed-back control $[123,124]$. However, in environments where external loads vary in time, direction and point of action, it will be very hard for soft actuators to outperform conventional stiff robots. 
More recently, the focus shifted from implementing software intelligence to hardware intelligence, where nonlinear actuator characteristics are tailored to a specific task [13]. For instance, tapered bending actuator curl-up when pressurised which allows to grab and translate objects via a single pressure input $[42,73]$. Further, the soft robotic field is embracing new nonlinear mechanisms that enrich their characteristics, like buckling of elastomeric beams [125], snap-through instabilities [126], nonlinear passive valves [127, 128] etc. This approach embeds advanced functionality in the hardware of the soft robot, but is far from established and is still a topic of ongoing research.

\section{Applications}

The shift from rigid to flexible actuators requires rethinking of how robots can contribute to industry and society. Obvious advantages of soft robots include their intrinsic safety, ability to mimic biologic systems and low cost. However, it is still unclear in which domains soft robotic actuators will displace existing rigid robot solutions or introduce new disruptive applications. So far, seven distinct applications domains have emerged in literature, some of which have found real commercial implementation. These application domains are illustrated on the timeline of Fig. 6 , and will be discussed in what follows.

\subsection{Automation}

Arguably, the most important application for classic rigid robots is automation where they perform tasks such as assembly and welding in production lines. Soft robotic actuators are unlikely to compete with this established technology but might complement these systems for instance in applications where compliant soft grippers are desired that deform around delicate objects (e.g. enclosing without contact before securing the object). This essentially bypasses the need for sensing and complex control algorithms. Soft robotic grippers were pioneered by James I. Baer, who in 1967 filed a patent [56] for a material handling apparatus which is capable of gripping objects by curling around them. The design of this gripper concept evolved over the following years to eliminate lateral movements in 1972 [129]. Further, more specific military and aerospace applications were pursued by Orndorff et al. [130] in 1971 and by Craig et al. [131] in 1989. Bridgestone et al. [132] (1984) on the other hand thought of a EIA gripper as a generalized robotic end-effector. Together with the increasing scientific interest in these actuators came a clear distinction between large scale grippers (> $10 \mathrm{~mm}$ ) and small scale grippers $(<10 \mathrm{~mm})$. Initially focusing on large grippers, Suzumori et al. $[39,133]$ reported in 1991 on the development of four threechambered actuators, each $12 \mathrm{~mm}$ in diameter, to pick up centimetre-sized objects and differentiated between pinching and grasping, which was theoretically analysed by mechanical models. Two of these three-chambered actuators were placed in front of a pipeinspection robot [134] (1999) making it possible to grasp objects ranging from 1 to $4 \mathrm{~mm}$ in size and 1 to $3 \mathrm{~g}$ in weight in about $0.5 \mathrm{~s}$. Similar to Suzumori et al.'s 1991-configuration, a four-fingered gripper was suggested by Udupa et al. [70] in 2010 to interact with living organisms in a safe way. Subsequently, Ilievski et al. [63] successfully picked up an uncooked 
chicken egg and an anesthetized mouse using a starfish-based gripper [135]. Soft robotics Inc. (2013) is commercializing these grippers for applications in warehouse logistics, advanced assembly and food handling, and other applications where different shapes need to be grasped using a single gripper [136]. In 2015, the company Materialise NV started activity in the same field [105] using 3D printed grippers. Recently, Hwang et al. [79] introduced a serial arrangement of bending actuators between a base and a three-fingered gripper, making positioning of the gripper in space possible. Further, Galloway et al. [137] (2016) demonstrated deep sea marine sample collection and manipulation with two pairs of opposing soft robotic grippers, without damaging red soft coral. Wei et al. [138] augmented a three-fingered gripper with a parallel particle jamming chamber to achieve a conformal gripping contact.

On a smaller scale $(<10 \mathrm{~mm})$, CJ Kim et al. $[64,139,140](2003)$ used a four-fingered gripper for manipulating biological tissue (capelin eggs, a nerve bundle, fatty tissue), where each finger could hold $0.2 \mathrm{~g}$ of weight. This gripper was equipped with a system allowing for visual system identification before grasping [141]. Similar two- and three-fingered concepts were explored by Kang et al. [26] (2006), Yoshida et al. [49] (2015) and Konishi et al. [142] (2016), the latter focussed on biocompatibility and surface treatment of the grippers to facilitate grasping of cell aggregates. Wakimoto et al. [24, 143] (2009) incorporated three bending actuators with corrugated membranes in a microgripper system which opens by applying vacuum, facilitating the grasping of fish eggs. In a different microgripper concept, Paek et al. [43] (2016) used a single spiralling tentacle to grasps biological tissue (mallotus villosus egg), similar to the large scale spiralling gripper of Galloway et al. [137] (2016) for gripping red soft coral.

In another automation application, Suzumori et al. [59] (1994) placed multiple single bending actuators parallel to each other to form a conveyor array. Similarly, Konishi et al. [112] (2001) strategically placed 16 bending EIAs in an array to incrementally move substrates.

The opportunities offered by EIA soft robotic manipulators to position an end-effector in 3D space have been explored extensively in literature. Suzumori et al. [12] (1991) developed a 3 DOF manipulator consisting of 3 radial chambers in a cylindrical tube, as a building block, to make a 7 DOF gripper (2 times a 3 DOF manipulator in series with a pneumatic gripper at its end-effector). The positioning performance of a single 3 DOF ( 2 bending and 1 extension) building block has been studied extensively by Ferraresi et al. [144] (1997). Martinez et al. [73] (2012) combined similar building blocks to form a 9 DOF manipulator (3 times a 3 DOF actuator). Wilson et al. [40] (2007) combined 5 bending EIAs and demonstrated pick and place capabilities. The company Festo on the other hand introduced its bionic handling assistant [145], a serial connection of 3 DOF actuators for applications where safe humanmachine contact is important. Marchese et al. investigated 2D robotic arms [74] (2014) by combining six two-chambered actuators with a total of 12 DOFs which showed both control of the end-effector as control of the entire arm, having the ability to manipulate in a 
confined pipe-like environment. In addition, Marchese et al. [75] (2015) introduced a four radial chamber actuator concept, and combined them in a 12 DOF robotic arm.

\subsection{Biomimetics}

Taking the idea of robotic grippers further, artificial robotic hands have been developed using EIA technology. This was reported for the first time in 1971 by Rich et al. who configured 5 bending EIAs in a hand pattern [34]. These fingers were consequently improved by Andorf et al. [146], in the so-called SIMRIT finger design. 20 years later, in 1996, Suzumori et al. [45] proposed a life-size hand with 5 actuated fingers to achieve hand-like grasping. On a smaller, millimetre scale, Konishi et al. [14, 62, 147, 148] (2005) developed a five-fingered microhand, controlled by a data glove, successfully realizing hybrid motion and manipulation of a single fish's egg and a hair. In a fibre-constrained design, Yamaguchi et al. $[22,66](2011)$ used five ECF-powered EIA fingers and devised actuation methodologies according to Cutkosky's human grasp taxonomy. Deimel et al. [88] (2013) successfully grasped different objects with a variety of weights and sizes using a static thumb. However this design was altered to an active five-fingered hand [149] in 2016 which closely mimics human hand motions. The 36 grasps of the Feix grasp taxonomy were enacted using empirically determined actuation patterns. In a fully planar design, Niiyama et al. [107, 108] $(2014)$ incorporated two actuator joints at each of the five fingers of their design, together with an articulated palm.

Bending EIAs have been used to actuate robotic legs for locomotion on land. The conveyer systems discussed above were turned upside down [45] to form a walking platform propelled by soft robotic legs with length of $15 \mathrm{~mm}$ and weight of $1 \mathrm{~g}$ that can walk at a speed of $200 \mathrm{~mm} / \mathrm{min}$ (i.e. 800 body lengths per hour) and has a load capacity of $300 \mathrm{mgf}$. This design was mimicked by Wilson et al. [40] in 2007, who used 1 DOF actuators instead of 3 DOF actuators. A multigait robot is proposed by Shepherd et al. [80, 135, 150, 151] (2011), that is composed only out of soft materials and uses 5 bending actuators. With a length of $13 \mathrm{~cm}$, this robot showed the ability to move at different gaits, with speeds between 93 and 192 body lengths per hour. Further, the design of this robot can be augmented with a camouflage layer [152] and be made resilient to adverse environmental conditions [99] and mechanical damage [153]. Further, these robots have been made sufficiently large to carry their own miniature compressors and pneumatic controls, allowing for untethered operation. Shepherd et al. [21] (2013) also fabricated a three-limb jumping robot, where each limb measures $75 \mathrm{~mm}$ in length and the driving pressure is generated by combusting methane. This robot is able to jump more than $30 \mathrm{~cm}$ high. Further iterations [81] on this design increased jumping height to $60 \mathrm{~cm}$ and rendered the robot untethered. In a design similar to the multigait robot, Stokes et al. [154] (2014) devised a hybrid robot that uses a hard robotic base with a tethered soft robotic trunk. Besides using bending actuators as robot limbs, others means of locomotion on land have been developed using soft actuators. A rolling robot with on-board chemical pressure generation was presented by Onal et al. [84] (2011), where internal bending actuators were placed on the surface of a wheel to 
incrementally push it further with a rolling step of $40 \mathrm{~mm}$. Onal et al. [155] (2013) proposed a serpentine inspired locomotion using four bidirectional bending actuators, each consisting of 2 parallel pressure chambers. Crawling locomotion is also reported by Correll et al. [83] (2014), who used a traveling wave over the length of the system body. Lastly, Waynelovich et al. [156] (2015) made an untethered robot that could walk for several hours.

In addition, EIAs are suitable for biomimetic propulsion in water. For instance, the swimming motion of manta's was imitated by Suzumori et al. [50] (2007) by using two 2DOFactuators and this system was able to achieve a swimming speed of $100 \mathrm{~mm} / \mathrm{s}$ in water. In another design inspired by nature, Kato et al. [52] (2008) created an artificial fish with pectoral fins consisting of three bending actuators, allowing for a flapping motion to swim. Aswath et al. [95] (2014) used two bending actuators protruding from a fish body, to mimic pectoral fins. Zhang et al. [53] and Marchese et al. [76, 77] (2014) designed a caudal fin actuated by 2 DOF bending EIAs. The latter integrated this fin actuator in an untethered fish robot with a length of $339 \mathrm{~mm}$, reaching swimming speeds of $150 \mathrm{~mm} / \mathrm{s}$, while having full control of speed, yaw and pitch. On a smaller scale, six bending actuators with length of $8 \mathrm{~mm}$ were combined to form a cilia array by Gorissen et al. [20] (2015), where each cilium is able to beat at frequencies of up to $35 \mathrm{~Hz}$. The cilia array is able to propel water with control over flow direction by changing the cilia beating pattern.

\subsection{Medical applications}

Because of their inherent safety [4], elastic inflatable actuators are particularly suitable for medical applications [157]. On the one hand, these applications can be non-invasive, such as an active support splint to assist wrist motions developed by Sasaki et al. [65] (2005) and a robotic glove to augment hand rehabilitation for functional grasp pathologies by Polygerinos et al. [16] (2015) and Yap et al. [106] (2016). On the other hand, invasive surgical applications for ElAs have attracted considerable attention since 1996 when Lazeroms et al. [47] suggested to use bending EIAs as a minimally invasive hydraulic forceps with forcefeedback. Konishi et al. [158] (2002) developed an actuator with integrated electrically conductive paths, forming a cuff actuator that can wrap around nerves for reliable electrical nerve stimulation. At the same time, Ikuta et al. [60, 127, 159] (2002) fabricated a catheterization tool with local degrees of freedom, consisting of two single chamber bending actuators in series that can be selectively actuated using intermediate bandpass valves. By refining the design, the world's smallest active catheter, with a diameter of $0.4 \mathrm{~mm}$ was developed [25]. Further, a method to reduce radial expansion during actuation, which is detrimental for manipulation in small veins, has been developed [96]. An alternative catheterization procedure was proposed by Haga et al. [68] (2005), who used an initially curved actuator that straightens by applying a negative pressure. In the ophthalmological domain, a minimally invasive surgical tool for retinal pigment epithelium transplantation was developed by Konishi et al. [90] (2007), consisting of two functional bending actuators, a suction sheet for loading a cell membrane and a tactile sensor [160]. The same research group utilized the bending deformation of multiple parallel actuators to operate surgical 
forceps [30] (2009) and reported on a retractor for spacing in front of an endoscope [161] (2011). Chen et al. [114] developed a "ColoBot" in 2009, a 3 DOF bending EIA to help with colon insertion. In 2013, a general abdominal minimally invasive surgical manipulator was developed using a 3DOF bending actuator, with an internal granular jamming mechanism to lock the actuator into shape [36, 72]. A similar granular design was reported in [136] (2015), where a surgical tool was positioned at the tip. Lastly, Konishi et al. [162] (2016) integrated an optical waveguide in soft actuators allowing for endoscopic imaging.

\section{Outlook}

Given the recent booming amount of research papers published on elastic inflatable actuators, it is believed that these will play a prominent role in the future of robotics. Thanks to their large strokes, complex motions and flexibility, they allow for a new generation of robots that are soft and complement existing rigid robotic technologies. Soft robots are especially interesting for tasks where safety, dexterity, and conformal deformation are important. Further, their ability to mimic biological systems and their low cost are important assets to give way to new, yet to discover application domains. However, several issues still need to be resolved in order for EIAs to be deployed more widely in robotic applications. The most important of these challenges are described below.

From a practical point of view, it is almost impossible to supply each actuator segment with its own pressure tubing when many actuators are combined to create a robot. Especially in the case of small scale systems, tubing can easily take up more space than the actual system. Except for a few exceptions [76, 81, 99, 111], ElA-based robots are always tethered and connected to a large external pressure source. Developing on-board pressure generation together with local valves are amongst the key problems to be solved for autonomous soft robotic systems. Solutions for this problem have been suggested in literature, using electrolysis of water [163], using electro-conjugate fluids [164-166], using chemical reactions [81], using a commercial electric pump [99], using gas stored under high pressure [76], using phase transformations [61, 167], using the decomposition of aqueous hydrogen peroxide [111], using an alternating pressure source and rectifying valves [93], using electrorheological valves [49], using micromachined valves [168], using bandpass valves [60] etc. A problem with many of these solutions is that they require electrical power and are essentially replacing a pneumatic tether by an electric one. A comparative study for internal pneumatic energy sources, including microcompressors, compressed fluid and direct chemical reactions can be found in [169]. An outcome of this study is that no technology is superiorly better than others in terms of energy capacity, flow capacity and pressure capacity on all scales. A recent interesting development consists of matching non-linearities of the pressure supply to non-linearities in the actuator design which enables optimizing the overall system. This approach could be particularly powerful in combination with for example snap-through instabilities, where small volumetric changes lead to large overall deformations $[125,126,170,171]$. 
The elastic materials EIAs are composed of, give rise to unique features such as safety and large deformations at low pressures, however these materials are also the source of several limitations. Firstly, the elasticity of the actuators inherently limits the actuation force they can exert. A solution to this problem was achieved by incorporation a channel inside the actuator that can change its stiffness via granular jamming [36] or via solidifying a lowmelting-point-alloy [172]. However, only limited stiffness variations have been achieved for large-scale actuators. Secondly, these soft materials are in general susceptible to damage caused by sharp objects, which leads to an inability to build up pressure and results in a loss of functionality. Even though punctured actuators remain inherently safe and become backdriveable, systems are developed that are more resilient to mechanical damage [153] or have a mechanical shielding [173], while other research groups reported on compatible selfsealing [82] and self-healing [174] materials Thirdly, these elastic materials have non-linear stress-strain characteristics making it particularly challenging to simulate and optimise these actuators as discussed above.

New nonlinear optimisation tools will allow for the development of an entirely new generation of soft robots with increasingly complex structures. To implement these designs, new easily accessible manufacturing techniques need to be developed that can create intricate 3D inflatable voids in highly elastic materials. These soft robotic systems will be able to perform advanced tasks without the need of complex control systems and will be characterized by continuous soft structures that simultaneously fulfil the role of robotic link and actuator. These new robots are anticipated to take a prominent role in delicate tasks where classic robots fail, such as in minimally invasive surgery, active prosthetics and automation tasks involving delicate irregular objects. Finally, because soft robots are radically different from existing stiff robots, they may enable entirely new application domains that are yet to be discovered.

\section{Acknowledgement}

This research is supported by the Fund for Scientific Research-Flanders (FWO), and the European Research Council (ERC starting grant HIENA). 


\section{References}

[1] Y. Yamada, Y. Hirasawa, S.Y. Huang, Y. Umetani, K. Suita, Human-robot contact in the safeguarding space, leee-Asme Transactions on Mechatronics 2(4) (1997) 230-236.

[2] D. Rus, M.T. Tolley, Design, fabrication and control of soft robots, Nature 521(7553) (2015) 467475.

[3] M. De Volder, D. Reynaerts, Pneumatic and hydraulic microactuators: a review, Journal of Micromechanics and Microengineering 20(4) (2010).

[4] A. De Greef, P. Lambert, A. Delchambre, Towards flexible medical instruments: Review of flexible fluidic actuators, Precision Engineering-Journal of the International Societies for Precision Engineering and Nanotechnology 33(4) (2009) 311-321.

[5] S. Kim, C. Laschi, B. Trimmer, Soft robotics: a bioinspired evolution in robotics, Trends in Biotechnology 31(5) (2013) 23-30.

[6] L.A. Fleury, Pneumatic jack, US1295471, 1919.

[7] B. Gorissen, T. Chishiro, S. Shimomura, D. Reynaerts, M. De Volder, S. Konishi, Flexible pneumatic twisting actuators and their application to tilting micromirrors, Sensors and Actuators a-Physical 216 (2014) 426-431.

[8] S. Hirai, P. Cusin, H. Tanigawa, T. Masui, S. Konishi, S. Kawamura, I. leee, Qualitative synthesis of deformable cylindrical actuators through constraint topology, 2000 leee/Rsj International Conference on Intelligent Robots and Systems (Iros 2000), Vols 1-3, Proceedings (2000) 197-202.

[9] S. Hirai, T. Masui, S. Kawamura, leee, I. leee, Prototyping pneumatic group actuators composed of multiple single-motion elastic tubes, IEEE International Conference on Robotics and Automation, Seoul, South Korea, 2001, pp. 3807-3812.

[10] J. Bishop-Moser, G. Krishnan, C. Kim, S. Kota, I.R.S.o. Japan, Design of Soft Robotic Actuators using Fluid-filled Fiber-Reinforced Elastomeric Enclosures in Parallel Combinations, 25th IEEE\RSJ International Conference on Intelligent Robots and Systems (IROS), Algarve, PORTUGAL, 2012, pp. 4264-4269.

[11] C.P. Chou, B. Hannaford, Measurement and modeling of McKibben pneumatic artificial muscles, leee Transactions on Robotics and Automation 12(1) (1996) 90-102.

[12] K. Suzumori, S. likura, H. Tanaka, leee, FLEXIBLE MICROACTUATOR FOR MINIATURE ROBOTS, leee Micro Electro Mechanical Systems: an Investigation of Micro Structures, Sensors, Actuators, Machines and Robots (1991) 204-209.

[13] F. Connolly, P. Polygerinos, C.J. Walsh, K. Bertoldi, Mechanical Programming of Soft Actuators by Varying Fiber Angle, Soft Robotics 2(1) (2015) 26-32.

[14] O.C. Jeong, S. Konishi, All PDMS pneumatic microfinger with bidirectional motion and its application, Journal of Microelectromechanical Systems 15(4) (2006) 896-903.

[15] B. Gorissen, M. De Volder, A. De Greef, D. Reynaerts, Theoretical and experimental analysis of pneumatic balloon microactuators, Sensors and Actuators a-Physical 168(1) (2011) 58-65.

[16] P. Polygerinos, Z. Wang, K.C. Galloway, R.J. Wood, C.J. Walsh, Soft robotic glove for combined assistance and at-home rehabilitation, Robotics and Autonomous Systems 73 (2015) 135-143.

[17] X. Gong, K. Yang, J. Xie, Y. Wang, P. Kulkarni, A.S. Hobbs, A.D. Mazzeo, Rotary Actuators Based on Pneumatically Driven Elastomeric Structures, Advanced Materials (2016) n/a-n/a.

[18] J.C. Case, E.L. White, R.K. Kramer, Soft Material Characterization for Robotic Applications, Soft Robotics 2(2) (2015) 80-87.

[19] B. Mosadegh, P. Polygerinos, C. Keplinger, S. Wennstedt, R.F. Shepherd, U. Gupta, J. Shim, K. Bertoldi, C.J. Walsh, G.M. Whitesides, Pneumatic Networks for Soft Robotics that Actuate Rapidly, Advanced Functional Materials 24(15) (2014) 2163-2170.

[20] B. Gorissen, M. de Volder, D. Reynaerts, Pneumatically-actuated artificial cilia array for biomimetic fluid propulsion, Lab on a Chip 15(22) (2015) 4348-4355.

[21] R.F. Shepherd, A.A. Stokes, J. Freake, J. Barber, P.W. Snyder, A.D. Mazzeo, L. Cademartiri, S.A. Morin, G.M. Whitesides, Using Explosions to Power a Soft Robot, Angewandte Chemie-International Edition 52(10) (2013) 2892-2896. 
[22] A. Yamaguchi, K. Takemura, S. Yokota, K. Edamura, A robot hand using electro-conjugate fluid, Sensors and Actuators a-Physical 170(1-2) (2011) 139-146.

[23] R.V. Martinez, C.R. Fish, X. Chen, G.M. Whitesides, Elastomeric Origami: Programmable PaperElastomer Composites as Pneumatic Actuators, Advanced Functional Materials 22(7) (2012) 13761384.

[24] S. Wakimoto, K. Ogura, K. Suzumori, Y. Nishioka, Miniature soft hand with curling rubber pneumatic actuators, 2009 IEEE International Conference on Robotics and Automation (ICRA) (2009) 556-61.

[25] M. Ikeuchi, K. Ikuta, leee, "Membrane micro emboss following excimer laser ablation (MEME-X) process" for pressure-driven micro active catheter, 21st IEEE International Conference on Micro Electro Mechanical Systems (MEMS 2008), Tucson, AZ, 2008, pp. 62-65.

[26] H.-W. Kang, I.H. Lee, D.-W. Cho, Development of a micro-bellows actuator using microstereolithography technology, Microelectronic Engineering 83(4-9) (2006) 1201-1204.

[27] K. Suzumori, T. Maeda, H. Watanabe, T. Hisada, Fiberless flexible microactuator designed by finite-element method, leee-Asme Transactions on Mechatronics 2(4) (1997) 281-286.

[28] O.C. Jeong, S. Kusuda, S. Konishi, leee, All PDMS pneumatic balloon actuators for bidirectional motion of micro finger, 18th IEEE International Conference on Micro Electro Mechanical Systems (MEMS), Miami Beach, FL, 2005, pp. 407-410.

[29] J. Hiller, H. Lipson, Automatic Design and Manufacture of Soft Robots, leee Transactions on Robotics 28(2) (2012) 457-466.

[30] N. Fujiwara, S. Sawano, S. Konishi, Linear expansion and contraction of paired pneumatic balloon bending actuators toward telescopic motion, 2009 IEEE 22nd International Conference on Micro Electro Mechanical Systems. MEMS 2009 (2009) 435-8.

[31] K. Morimoto, A. Utsumi, S. Konishi, A design of longitudinally-divided balloon structure in PDMS pneumatic balloon actuator based on fem simulations, 2011 16th International Solid-State Sensors, Actuators and Microsystems Conference, 2011, pp. 2774-2777.

[32] A.D. Marchese, R.K. Katzschmann, D. Rus, A Recipe for Soft Fluidic Elastomer Robots, Soft Robotics 2(1) (2015) 7-25.

[33] L. Zentner, V. Boehm, V. Minchenya, On the new reversal effect in monolithic compliant bending mechanisms with fluid driven actuators, Mechanism and Machine Theory 44(5) (2009) 1009-1018.

[34] S.R. Rich, Fluid operable motor, US3561330, 1971.

[35] K. Ogura, S. Wakimoto, K. Suzumori, Y. Nishioka, leee, Micro Pneumatic Curling Actuator Nematode Actuator, 2008 leee International Conference on Robotics and Biomimetics, Vols 1-4 (2009) 462-467.

[36] M. Cianchetti, T. Ranzani, G. Gerboni, I. De Falco, C. Laschi, A. Menciassi, STIFF-FLOP Surgical Manipulator: mechanical design and experimental characterization of the single module, IEEE/RSJ International Conference on Intelligent Robots and Systems (IROS), Tokyo, JAPAN, 2013, pp. 35763581.

[37] L. Zentner, S. Petkun, R. Blickhan, From the spider leg to a hydraulic device, Technische mechanik 20(1) (1999).

[38] Y. Shapiro, A. Wolf, K. Gabor, Bi-bellows: Pneumatic bending actuator, Sensors and Actuators aPhysical 167(2) (2011) 484-494.

[39] K. Suzumori, S. likura, H. Tanaka, Development of flexible microactuator and its applications to robotic mechanisms, Proceedings. 1991 IEEE International Conference on Robotics and Automation (Cat. No.91CH2969-4) (1991) 1622-7 vol.2.

[40] J.F. Wilson, N. Inou, Bellows-type springs for robotics, Advanced Spring Technology JSSE 60th Anniversary Int. Symposium, Nagoya, Japan, 2007, pp. 109-119.

[41] B. Gorissen, W. Vincentie, F. Al-Bender, D. Reynaerts, M. De Volder, Modeling and bonding-free fabrication of flexible fluidic microactuators with a bending motion, Journal of Micromechanics and Microengineering 23(4) (2013).

[42] X. Liang, Y. Sun, H. Ren, A Flexible Fabrication Approach towards the Shape Engineering of Microscale Soft Pneumatic Actuators, IEEE Robotics and Automation Letters PP(99) (2016) 1-1. 
[43] J. Paek, I. Cho, J. Kim, Microrobotic tentacles with spiral bending capability based on shapeengineered elastomeric microtubes, Scientific Reports 5 (2015).

[44] K.M. de Payrebrune, O.M. O'Reilly, On constitutive relations for a rod-based model of a pneu-net bending actuator, Extreme Mechanics Letters.

[45] K. Suzumori, Elastic materials producing compliant robots, Robotics and Autonomous Systems 18(1-2) (1996) 135-140.

[46] J. Fras, J. Czarnowski, M. Macias, J. Glowka, Static Modeling of Multisection Soft Continuum Manipulator for Stiff-Flop Project, Recent Advances in Automation, Robotics and Measuring Techniques 267 (2014) 365-375.

[47] M. Lazeroms, A. LaHaye, W. Sjoerdsma, W. Schreurs, W. Jongkind, G. Honderd, C. Grimbergen, A hydraulic forceps with force-feedback for use in minimally invasive surgery, Mechatronics 6(4) (1996) 437-446.

[48] K. Suzumori, S. likura, H. Tanaka, Applying a flexible microactuator to robotic mechanisms, IEEE Control Systems Magazine 12(1) (1992) 21-7.

[49] K. YOSHIDA, S. HARA, S.I. EOM, S. YOKOTA, A novel soft microgripper using divided-electrode type flexible ER valves, Mechanical Engineering Journal (2015).

[50] K. Suzumori, S. Endo, T. Kanda, N. Kato, H. Suzuki, leee, A bending pneumatic rubber actuator realizing soft-bodied manta swimming robot, IEEE International Conference on Robotics and Automation, Rome, ITALY, 2007, pp. 4975-4980.

[51] B. Kim, S.B. Lee, J. Lee, S. Cho, H. Park, S. Yeom, S.H. Park, A comparison among Neo-Hookean model, Mooney-Rivlin model, and Ogden model for chloroprene rubber, International Journal of Precision Engineering and Manufacturing 13(5) (2012) 759-764.

[52] N. Kato, Y. Ando, A. Tomokazu, H. Suzuki, K. Suzumori, T. Kanda, S. Endo, Elastic pectoral fin actuators for biomimetic underwater vehicles, Bio-Mechanisms of Swimming and Flying: Fluid Dynamics, Biomimetic Robots, and Sports Science (2008) 271-282.

[53] J.H. Zhang, J.Q. Tang, J. Hong, T.Q. Lu, H. Wang, The Design and Analysis of Pneumatic Rubber Actuator of Soft Robotic Fish, Intelligent Robotics and Applications, Icira 2014, Pt I 8917 (2014) 320327.

[54] Y. Matia, A.D. Gat, Dynamics of Elastic Beams with Embedded Fluid-Filled Parallel-Channel Networks, Soft Robotics 2(1) (2015) 42-47.

[55] H. Lipson, Challenges and Opportunities for Design, Simulation, and Fabrication of Soft Robots, Soft Robotics 1(1) (2014) 21-27.

[56] J.I. Baer, Material handling apparatus and the like, US3343864, 1967.

[57] K. Suzumori, Flexible Microactuator, Trans. of Japan Society of Mechanical engineers 55(518) (1989) 2547-2552.

[58] K. Suzumori, T. Matsumaru, S. likura, Elastically deformable fluid actuator, US4976191, 1990.

[59] K. Suzumori, A. Koga, H. Riyoko, Microfabrication of integrated FMAS using stereo lithography, Proceedings IEEE Micro Electro Mechanical Systems. An Investigation of Micro Structures, Sensors, Actuators, Machines and Robotic Systems (Cat. No.94CH3404-1) (1994) 136-41.

[60] K. Ikuta, H. Ichikawa, K. Suzuki, Safety-active catheter with multiple-segments driven by microhydraulic actuators, Medical Image Computing and Computer-Assisted Intervention-Miccai 2002, Pt 1 2488 (2002) 182-191.

[61] F. Kawai, P. Cusin, S. Konishi, Thin flexible end-effector using pneumatic balloon actuator, Micro Electro Mechanical Systems, 2000. MEMS 2000. The Thirteenth Annual International Conference on, 2000, pp. 391-396.

[62] S. Konishi, M. Nokata, O.C. Jeong, T. Sakakibara, S. Kusuda, M. Kuwayama, H. Tsutsumi, Merging micro and macro robotics toward micro manipulation for biomedical operation, The 36th International Symposium on Robotics, Tokyo, Japan, 2005.

[63] F. Ilievski, A.D. Mazzeo, R.E. Shepherd, X. Chen, G.M. Whitesides, Soft Robotics for Chemists, Angewandte Chemie-International Edition 50(8) (2011) 1890-1895. 
[64] Y. Lu, C.-J. Kim, Micro-finger articulation by pneumatic parylene balloons, TRANSDUCERS '03. 12th International Conference on Solid-State Sensors, Actuators and Microsystems. Digest of Technical Papers (Cat. No.03TH8664) (2003) 276-9 vol.1.

[65] D. Sasaki, T. Noritsugu, M. Takaiwa, leee, Development of active support splint driven by pneumatic soft actuator (ASSIST), IEEE International Conference on Robotics and Automation (ICRA), Barcelona, SPAIN, 2005, pp. 520-525.

[66] A. Yamaguchi, K. Takemura, S. Yokota, K. Edamura, A robot hand using electro-conjugate fluid: Grasping experiment with balloon actuators inducing a palm motion of robot hand, Sensors and Actuators a-Physical 174 (2012) 181-188.

[67] M. Memarian, R. Gorbet, D. Kulic, leee, Modelling and Experimental analysis of a Novel Design for Soft Pneumatic Artificial Muscles, IEEE/RSJ International Conference on Intelligent Robots and Systems (IROS), Hamburg, GERMANY, 2015, pp. 1718-1724.

[68] Y. Haga, Y. Muyari, T. Mineta, T. Matsunaga, H. Akahori, M. Esashi, leee, Small diameter hydraulic active bending catheter using laser processed super elastic alloy and silicone rubber tube, 3rd IEEE/EMBS Special Topic Conference on Microtechnology in Medicine and Biology, Oahu, HI, 2005, pp. 245-248.

[69] K. Suzumori, M. Mihara, S. Wakimoto, Beautiful Flexible Microactuator changing its structural color with variable pitch grating, Robotics and Automation (ICRA), 2011 IEEE International Conference on, 2011, pp. 2771-2776.

[70] G. Udupa, P. Sreedharan, K. Aditya, Robotic gripper driven by flexible microactuator based on an innovative technique, Proceedings 2010 IEEE Workshop on Advanced Robotics and its Social Impacts (ARSO 2010) (2010) 111-16.

[71] M. Schworer, M. Kohl, W. Menz, Fluidic microjoints based on spider legs, Actuator, Bremen, Germany, 1998.

[72] T. Ranzani, M. Cianchetti, G. Gerboni, I. De Falco, A. Menciassi, A Soft Modular Manipulator for Minimally Invasive Surgery: Design and Characterization of a Single Module, leee Transactions on Robotics 32(1) (2016) 187-200.

[73] R.V. Martinez, J.L. Branch, C.R. Fish, L. Jin, R.F. Shepherd, R.M.D. Nunes, Z. Suo, G.M. Whitesides, Robotic Tentacles with Three-Dimensional Mobility Based on Flexible Elastomers, Advanced Materials 25(2) (2013) 205-212.

[74] A.D. Marchese, R.K. Katzschmann, D. Rus, leee, Whole Arm Planning for a Soft and Highly Compliant 2D Robotic Manipulator, IEEE/RSJ International Conference on Intelligent Robots and Systems (IROS), Chicago, IL, 2014, pp. 554-560.

[75] A.D. Marchese, D. Rus, Design, kinematics, and control of a soft spatial fluidic elastomer manipulator, The International Journal of Robotics Research (2015).

[76] A.D. Marchese, C.D. Onal, D. Rus, Autonomous Soft Robotic Fish Capable of Escape Maneuvers Using Fluidic Elastomer Actuators, Soft Robotics 1(1) (2014) 75-87.

[77] R.K. Katzschmann, A.D. Marchese, D. Rus, Hydraulic Autonomous Soft Robotic Fish for 3D

Swimming Hydraulic Autonomous Soft Robotic Fish for 3D Swimming, International Symposium on Experimental Robotics (ISER), Marrakech, Morocco, 2014.

[78] M. Benjamin Chang and Allison Chew and Nastaran Naghshineh and Carlo, A spatial bending fluidic actuator: fabrication and quasi-static characteristics, Smart Materials and Structures 21(4) (2012) 045008.

[79] Y. Hwang, O.H. Paydar, R.N. Candler, Pneumatic microfinger with balloon fins for linear motion using 3D printed molds, Sensors and Actuators a-Physical 234 (2015) 65-71.

[80] R.F. Shepherd, F. Ilievski, W. Choi, S.A. Morin, A.A. Stokes, A.D. Mazzeo, X. Chen, M. Wang, G.M. Whitesides, Multigait soft robot, Proceedings of the National Academy of Sciences of the United States of America 108(51) (2011) 20400-20403.

[81] M.T. Tolley, R.F. Shepherd, M. Karpelson, N.W. Bartlett, K.C. Galloway, M. Wehner, R. Nunes, G.M. Whitesides, R.J. Wood, leee, An Untethered Jumping Soft Robot, IEEE/RSJ International Conference on Intelligent Robots and Systems (IROS), Chicago, IL, 2014, pp. 561-566. 
[82] R.F. Shepherd, A.A. Stokes, R.M.D. Nunes, G.M. Whitesides, Soft Machines That are Resistant to Puncture and That Self Seal, Advanced Materials 25(46) (2013) 6709-6713.

[83] N. Correll, Ç.D. Önal, H. Liang, E. Schoenfeld, D. Rus, Soft Autonomous Materials-Using Active Elasticity and Embedded Distributed Computation, in: O. Khatib, V. Kumar, G. Sukhatme (Eds.), Experimental Robotics: The 12th International Symposium on Experimental Robotics, Springer Berlin Heidelberg, Berlin, Heidelberg, 2014, pp. 227-240.

[84] C.D. Onal, X. Chen, G.M. Whitesides, D. Rus, Soft Mobile Robots with On-Board Chemical Pressure Generation, International Symposium on Robotics Research (ISRR), 2011.

[85] Y. Sun, Y.S. Song, J. Paik, Characterization of Silicone Rubber Based Soft Pneumatic Actuators, IEEE/RSJ International Conference on Intelligent Robots and Systems (IROS), Tokyo, JAPAN, 2013, pp. 4446-4453.

[86] A.D. Marchese, K. Komorowski, C.D. Onal, D. Rus, Design and control of a soft and continuously deformable 2D robotic manipulation system, 2014 IEEE International Conference on Robotics and Automation (ICRA) (2014) 2189-96.

[87] C.D. Onal, D. Rus, A Modular Approach to Soft Robots, 4th IEEE RAS and EMBS International Conference on Biomedical Robotics and Biomechatronics (BioRob) / Symposium on Surgical Robotics, Rome, ITALY, 2012, pp. 1038-1045.

[88] R. Deimel, O. Brock, leee, A Compliant Hand Based on a Novel Pneumatic Actuator, IEEE International Conference on Robotics and Automation (ICRA), Karlsruhe, GERMANY, 2013, pp. 20472053.

[89] Y.N. Xia, G.M. Whitesides, Soft lithography, Angewandte Chemie-International Edition 37(5) (1998) 550-575.

[90] Y. Watanabe, M. Maeda, N. Yaji, R. Nakamura, H. Iseki, M. Yamato, T. Okano, S. Hori, S. Konishi, leee, Small, soft, and safe microactuator for Retinal Pigment Epithelium transplantation, 20th IEEE International Conference on Micro Electro Mechanical Systems (MEMS 2007), Kobe, JAPAN, 2007, pp. 494-497.

[91] K. Yoshida, I. Toru, J.w. Kim, S. Yokota, A microgripper using electro-rheological fluid, ICCAS-SICE, 2009, 2009, pp. 2987-2990.

[92] T. Miyoshi, K. Yoshida, J.W. Kim, S.I. Eom, S. Yokota, Development of a MEMS-based electrorheological microfinger system with an alternating pressure source, 2015 Transducers - 2015 18th International Conference on Solid-State Sensors, Actuators and Microsystems (TRANSDUCERS), 2015, pp. 823-826.

[93] T. Miyoshi, K. Yoshida, J.-w. Kim, S.I. Eom, S. Yokota, An MEMS-based multiple electrorheological bending actuator system with an alternating pressure source, Sensors and Actuators A: Physical 245 (2016) 68-75.

[94] B. Gorissen, R. Donose, D. Reynaerts, M. De Volder, Flexible pneumatic micro-actuators: analysis and production, Procedia Engineering 25 (2011) 681-684.

[95] S. Aswath, C.K. Tilak, K. Jayaprakash, A.G. Nair, A.S. Nair, A. Mohan, G. Udupa, Innovative fish robot based on an asymmetrix flexible pneumatic actuator, International journal of advances in mechanical and automobile engineering 1(1) (2014) 101-107.

[96] M. Ikeuchi, K. Ikuta, leee, Development of Pressure-Driven Micro Active Catheter using Membrane Micro Emboss Following Excimer Laser Ablation (MeME-X) Process, IEEE International Conference on Robotics and Automation, Kobe, JAPAN, 2009, pp. 4358-4361.

[97] M.P. Benjamin Che-Ming Chang and John Berring and Manu Venkataram and Carlo Menon and, Bending fluidic actuator for smart structures, Smart Materials and Structures 20(3) (2011) 035012.

[98] T. Giffney, M. Xie, A. Yong, A. Wong, P. Mousset, A. McDaid, K. Aw, Soft Pneumatic Bending Actuator with Integrated Carbon Nanotube Displacement Sensor, Robotics 5(1) (2016).

[99] M.T. Tolley, R.F. Shepherd, B. Mosadegh, K.C. Galloway, M. Wehner, M. Karpelson, R.J. Wood, G.M. Whitesides, A Resilient, Untethered Soft Robot, Soft Robotics 1(3) (2014) 213-223.

[100] B. Gorissen, C. Van Hoof, D. Reynaerts, M. De Volder, SU8 etch mask for patterning PDMS and its application to flexible fluidic microactuators, Microsystems \& nanoengineering 2 (2016). 
[101] B.N. Peele, T.J. Wallin, H.C. Zhao, R.F. Shepherd, 3D printing antagonistic systems of artificial muscle using projection stereolithography, Bioinspiration \& Biomimetics 10(5) (2015).

[102] Y. Shapiro, A. Wolf, G. Kosa, Piezoelectric Deflection Sensor for a Bi-Bellows Actuator, leeeAsme Transactions on Mechatronics 18(3) (2013) 1226-1230.

[103] Y. Shapiro, K. Gabor, A. Wolf, Modeling a Hyperflexible Planar Bending Actuator as an Inextensible Euler-Bernoulli Beam for Use in Flexible Robots, Soft Robotics 2(2) (2015) 71-79.

[104] D. Han, H. Gu, J.-w. Kim, S. Yokota, K. Edamura, A Novel 3D-Printed Finger Integrated with ECF Micropump, ICMDT2015 Proceedings (No.) (2015) 124 - 125.

[105] P. Schiettecatte, R. Plaghki, Gripping apparatus and method of manufacturing a gripping apparatus, WO2014131810A1, 2014.

[106] H.K. Yap, H.Y. Ng, C.-H. Yeow, High-Force Soft Printable Pneumatics for Soft Robotic Applications, Soft Robotics 3(3) (2016) 144-158.

[107] R. Niiyama, X. Sun, C. Sung, B. An, D. Rus, S. Kim, Pouch Motors: Printable Soft Actuators Integrated with Computational Design, Soft Robotics 2(2) (2015) 59-70.

[108] R. Niiyama, D. Rus, K. Sangbae, Pouch motors: printable/inflatable soft actuators for robotics, 2014 IEEE International Conference on Robotics and Automation (ICRA) (2014) 6332-7.

[109] B.C. Mac Murray, X. An, S.S. Robinson, I.M. van Meerbeek, K.W. O'Brien, H.C. Zhao, R.F. Shepherd, Poroelastic Foams for Simple Fabrication of Complex Soft Robots, Advanced Materials 27(41) (2015) 6334-+.

[110] A. Argiolas, B.C. Mac Murray, I. Van Meerbeek, J. Whitehead, E. Sinibaldi, B. Mazzolai, R.F. Shepherd, Sculpting Soft Machines, Soft Robotics (2016).

[111] M. Wehner, R.L. Truby, D.J. Fitzgerald, B. Mosadegh, G.M. Whitesides, J.A. Lewis, R.J. Wood, An integrated design and fabrication strategy for entirely soft, autonomous robots, Nature 536(7617) (2016) 451-5.

[112] S. Konishi, F. Kawai, P. Cusin, Thin flexible end-effector using pneumatic balloon actuator, Sensors and Actuators a-Physical 89(1-2) (2001) 28-35.

[113] G. Belforte, E. Gay, Identification of static nonlinearities in a deformable pneumatic actuator, Proceedings of the 38th IEEE Conference on Decision and Control (Cat. No.99CH36304) (1999) 32023 vol.4.

[114] G. Chen, M.T. Pham, T. Redarce, Sensor-based guidance control of a continuum robot for a semi-autonomous colonoscopy, Robotics and Autonomous Systems 57(6-7) (2009) 712-722.

[115] K. Kure, T. Kanda, K. Suzumori, S. Wakimoto, Flexible displacement sensor using injected conductive paste, Sensors and Actuators a-Physical 143(2) (2008) 272-278.

[116] X. Wang, T. Geng, Y. Elsayed, T. Ranzani, C. Saaj, C. Lekakou, leee, A new coefficient-adaptive orthonormal basis function model structure for identifying a class of pneumatic soft actuators, IEEE/RSJ International Conference on Intelligent Robots and Systems (IROS), Chicago, IL, 2014, pp. 530-535.

[117] G. Belforte, F. Dabbene, P. Gay, Sensor design, identification and control of a deformable pneumatic actuator, International Journal of Mechanics and Control 4(1) (2003).

[118] G. Belforte, F. Dabbene, C. Ferraresi, P. Gay, A.M. Bertetto, Sensorizzazione e controllo di un manipolatore pneumatico flessibile, Convegno Nazionale AIAS, 2001.

[119] G. Belforte, F. Dabbene, P. Gay, Optimal design of a measurement system for the calibration of a flexible pneumatic actuator, Proceedings of the 1998 IEEE International Conference on Control Applications (Cat. No.98CH36104) (1998) 532-6 vol.1.

[120] J.A. LESSING, R.V. Martinez, A.S. TAYI, J.M. Ting, G.M. Whitesides, Flexible and stretchable electronic strain-limited layer for soft actuators, WO2016029130A1, 2016.

[121] Y. Yamamoto, K. Kure, T. Iwai, T. Kanda, K. Suzumori, Flexible displacement sensor using piezoelectric polymer for intelligent FMA, 2007 IEEE/RSJ International Conference on Intelligent Robots and Systems (2007) 765-70.

[122] S. Kusuda, S. Sawano, S. Konishi, Fluid-resistive bending sensor having perfect compatibility with flexible pneumatic balloon actuator, 2007 20th IEEE International Conference on Micro Electro Mechanical Systems - MEMS '07 (2007) 615-18. 
[123] M. De Volder, A.J.M. Moers, D. Reynaerts, Fabrication and control of miniature McKibben actuators, Sensors and Actuators a-Physical 166(1) (2011) 111-116.

[124] M. De Volder, J. Coosemans, R. Puers, D. Reynaerts, Characterization and control of a pneumatic microactuator with an integrated inductive position sensor, Sensors \& Actuators: A. Physical (2008) 192-200.

[125] D. Yang, B. Mosadegh, A. Ainla, B. Lee, F. Khashai, Z.G. Suo, K. Bertoldi, G.M. Whitesides, Buckling of Elastomeric Beams Enables Actuation of Soft Machines, Advanced Materials 27(41) (2015) 6323-+.

[126] J.T.B. Overvelde, T. Kloek, J.J.A. D'Haen, K. Bertoldi, Amplifying the response of soft actuators by harnessing snap-through instabilities, Proceedings of the National Academy of Sciences of the United States of America 112(35) (2015) 10863-10868.

[127] K. Ikuta, H. Ichikawa, K. Suzuki, D. Yajima, Multi-degree of freedom hydraulic pressure driven safety active catheter, Proceedings. 2006 Conference on International Robotics and Automation (IEEE Cat. No. 06CH37729D) (2006) 4161-6.

[128] N. Napp, B. Araki, M.T. Tolley, R. Nagpal, R.J. Wood, leee, Simple Passive Valves for Addressable Pneumatic Actuation, IEEE International Conference on Robotics and Automation (ICRA), Hong Kong, PEOPLES R CHINA, 2014, pp. 1440-1445.

[129] H.L. Kuster, Fluid-operated actuator, US3640564, 1972.

[130] R.L. Orndorff, Gripping device, US3601442, 1971.

[131] P.S. Craig, J.A. Fisher, Grappling device, US4815782, 1989.

[132] EPW, Rubber muscles take robotics one step further, Rubber Development 37(4) (1984) 117.

[133] K. Suzumori, Gripping actuator with independently flexible cylinders, US5156081, 1992.

[134] K. Suzumori, T. Miyagawa, M. Kimura, Y. Hasegawa, Micro inspection robot for 1-in pipes, leeeAsme Transactions on Mechatronics 4(3) (1999) 286-292.

[135] F. llievski, X. Chen, A.D. Mazzeo, G.M. Whitesides, R.F. Shepherd, R.V. Martinez, W.J. Choi, S.W. Kwok, S.A. Morin, A. Stokes, Soft robotic actuators, US20140109560, 2014.

[136] J.A. LESSING, R.R. KNOPF, N. Mclellan, Soft robotic actuators utilizing asymmetric surfaces, WO2015191585A1, 2015.

[137] K.C. Galloway, K.P. Becker, B. Phillips, J. Kirby, S. Licht, D. Tchernov, R.J. Wood, D.F. Gruber, Soft Robotic Grippers for Biological Sampling on Deep Reefs, Soft Robotics 3(1) (2016) 23-33.

[138] Y. Wei, Y. Chen, T. Ren, Q. Chen, C. Yan, Y. Yang, Y. Li, A Novel, Variable Stiffness Robotic Gripper Based on Integrated Soft Actuating and Particle Jamming, Soft Robotics (2016).

[139] Y.W. Lu, Z.Y. An, C.J. Kim, leee, A microhand: Modeling, manufacturing, and demonstration, 18th IEEE International Conference on Micro Electro Mechanical Systems (MEMS), Miami Beach, FL, 2005, pp. 650-653.

[140] Y.-W. Lu, C.-J. Kim, Microhand for biological applications, Applied Physics Letters 89(16) (2006). [141] W. Choi, M. Akbarian, V. Rubtsov, C.-J. Kim, Microhand With Internal Visual System, leee Transactions on Industrial Electronics 56(4) (2009) 1005-1011.

[142] S. Konishi, S. Shimomura, S. Tajima, Y. Tabata, Implementation of soft microfingers for a hMSC aggregate manipulation system, Microsystems \& Nanoengineering 2 (2016) 15048.

[143] S. Wakimoto, K. Suzumori, K. Ogura, Miniature Pneumatic Curling Rubber Actuator Generating Bidirectional Motion with One Air-Supply Tube, Advanced Robotics 25(9-10) (2011) 1311-1330.

[144] C. Ferraresi, L. Mazza, Design and Realisation of a Flexible Pneumatic Actuator for Robotics, 5th Scandinavian International Conference on Fluid Power, SICFP '97, Linkoping, 1997, pp. 29-43.

[145] G. Andrzej, B. Ralf, V. Alexander, The Bionic Handling Assistant: a success story of additive manufacturing, Assembly Automation 31(4) (2011) 329-333.

[146] P. Andorf, D. Franz, A. Lieb, G. Upper, W. Guttropf, Robot finger, US3981528, 1976.

[147] O.K. Jeong, S. Kusuda, T. Sakakibara, S. Konishi, M. Nokata, leee, Pneumatic micro finger as endeffecter of robot, Proceedings of the 2005 International Symposium on Micro-NanoMechatronics and Human Science: FROM MICRO \& NANO SCALE SYSTEMS TO ROBOTICS \& MECHATRONICS SYSTEMS (2005) 145-148. 
[148] S. Konishi, M. Nokata, J. Ok Chan, S. Kusuda, T. Sakakibara, M. Kuwayama, H. Tsutsumi, Pneumatic micro hand and miniaturized parallel link robot for micro manipulation robot system, Proceedings. 2006 Conference on International Robotics and Automation (IEEE Cat. No. 06CH37729D) (2006) 1036-41.

[149] R. Deimel, O. Brock, A novel type of compliant and underactuated robotic hand for dexterous grasping, International Journal of Robotics Research 35(1-3) (2016) 161-185.

[150] C. Majidi, R.F. Shepherd, R.K. Kramer, G.M. Whitesides, R.J. Wood, Influence of surface traction on soft robot undulation, International Journal of Robotics Research 32(13) (2013) 1577-1584.

[151] X. Zhou, C. Majidi, O.M. O'Reilly, Flexing into motion: A locomotion mechanism for soft robots, International Journal of Non-Linear Mechanics 74 (2015) 7-17.

[152] S.A. Morin, R.F. Shepherd, S.W. Kwok, A.A. Stokes, A. Nemiroski, G.M. Whitesides, Camouflage and Display for Soft Machines, Science 337(6096) (2012) 828-832.

[153] R.V. Martinez, A.C. Glavan, C. Keplinger, A.I. Oyetibo, G.M. Whitesides, Soft Actuators and Robots that Are Resistant to Mechanical Damage, Advanced Functional Materials 24(20) (2014) 30033010.

[154] A.A. Stokes, R.F. Shepherd, S.A. Morin, F. Ilievski, G.M. Whitesides, A Hybrid Combining Hard and Soft Robots, Soft Robotics 1(1) (2014) 70-74.

[155] C.D. Onal, D. Rus, Autonomous undulatory serpentine locomotion utilizing body dynamics of a fluidic soft robot, Bioinspiration \& Biomimetics 8(2) (2013).

[156] J. Waynelovich, T. Frey, A. Baljon, P. Salamon, Versatile and Dexterous Soft Robotic Leg System for Untethered Operations, Soft Robotics 3(2) (2016) 64-70.

[157] H.M. Le, T.N. Do, S.J. Phee, A survey on actuators-driven surgical robots, Sensors and Actuators A: Physical 247 (2016) 323-354.

[158] S. Konishi, T. Kobayashi, H. Maeda, S. Asajima, M. Makikawa, Cuff actuator for adaptive holding condition around nerves, Sensors and Actuators B-Chemical 83(1-3) (2002) 60-66.

[159] K. Ikuta, H. Ichikawa, K. Suzuki, T. Yamamoto, Safety active catheter with multi-segments driven by innovative hydro-pressure micro actuators, Micro Electro Mechanical Systems, 2003. MEMS-03

Kyoto. IEEE The Sixteenth Annual International Conference on, 2003, pp. 130-135.

[160] M. Tokida, T. Obara, M. Takahashi, M. Yamato, S. Konishi, leee, INTEGRATION OF CELL SHEET SUCKING AND TACTILE SENSING FUNCTIONS TO RETINAL PIGMENT EPITHELIUM TRANSPLANTATION TOOL, 23rd IEEE International Conference on Micro Electro Mechanical Systems (MEMS 2010), Hong Kong, PEOPLES R CHINA, 2010, pp. 316-319.

[161] S. Konishi, Small, soft, safe micromachine for minimally invasive surgery, 2011 International Meeting for Future of Electron Devices (IMFEDK) (2011) 20-1.

[162] S. Konishi, T. Kobayashi, Y. Muramatsu, Integration of optical waveguide on pneumatic balloon actuator for flexible scanner in endoscopic imaging diagnosis applications, Advanced Robotics 30(15) (2016) 1004-1013.

[163] K. Suzumori, A. Wada, S. Wakimoto, New mobile pressure control system for pneumatic actuators, using reversible chemical reactions of water, Sensors and Actuators a-Physical 201 (2013) 148-153.

[164] R. Abe, K. Takemura, K. Edamura, S. Yokota, Concept of a micro finger using electro-conjugate fluid and fabrication of a large model prototype, Sensors and Actuators a-Physical 136(2) (2007) 629637.

[165] J.-w. Kim, T. Suzuki, S. Yokota, K. Edamura, Tube-type micropump by using electro-conjugated fluid (ECF), Sensors and Actuators A: Physical 174 (2012) 155-161.

[166] J.-W. Kim, T.V.X. Nguyen, K. Edamura, S. Yokota, Triangular Prism and Slit Electrode Pair for ECF Jetting Fabricated by Thick Micromold and Electroforming as Micro Hydraulic Pressure Source for Soft Microrobots, International Journal of Automation Technology 10(No. 4) (2016) 470 - 478.

[167] S. Ogden, L. Klintberg, G. Thornell, K. Hjort, R. Boden, Review on miniaturized paraffin phase change actuators, valves, and pumps, Microfluidics and Nanofluidics 17(1) (2014) 53-71.

[168] A.J.M. Moers, M.F.L. De Volder, D. Reynaerts, Integrated high pressure microhydraulic actuation and control for surgical instruments, Biomedical Microdevices 14(4) (2012) 699-708. 
[169] M. Wehner, M.T. Tolley, Y. Menguc, Y.L. Park, A. Mozeika, Y. Ding, C. Onal, R.F. Shepherd, G.M. Whitesides, R.J. Wood, Pneumatic Energy Sources for Autonomous and Wearable Soft Robotics, Soft Robotics 1(4) (2014) 263-274.

[170] L. Hines, K. Petersen, M. Sitti, Inflated Soft Actuators with Reversible Stable Deformations, Advanced Materials 28(19) (2016) 3690-3696.

[171] D. Glozman, N. Hassidov, M. Senesh, M. Shoham, A Self-Propelled Inflatable Earthworm-Like Endoscope Actuated by Single Supply Line, leee Transactions on Biomedical Engineering 57(6) (2010) 1264-1272.

[172] Z. Lanying, S. Yoshida, Y. Morimoto, H. Onoe, S. Takeuchi, Pneumatic balloon actuator with tunable bending points, 2015 28th IEEE International Conference on Micro Electro Mechanical Systems (MEMS) (2015) 18-21.

[173] L. Paez, G. Agarwal, J. Paik, Design and Analysis of a Soft Pneumatic Actuator with Origami Shell Reinforcement, Soft Robotics (2016).

[174] S. Terryn, G. Mathijssen, J. Brancart, D. Lefeber, G. Van Assche, B. Vanderborght, Development of a self-healing soft pneumatic actuator: a first concept, Bioinspiration \& Biomimetics 10(4) (2015) 046007.

[175] C. Moraes, Y. Sun, C.A. Simmons, Solving the shrinkage-induced PDMS alignment registration issue in multilayer soft lithography, Journal of Micromechanics and Microengineering 19(6) (2009). [176] Y.C. Su, L.W. Lin, A.P. Pisano, A water-powered osmotic microactuator, Journal of Microelectromechanical Systems 11(6) (2002) 736-742.

[177] M.C. Birch, R.D. Quinn, G. Hahm, S.M. Phillips, B. Drennan, A. Fife, H. Verma, R.D. Beer, Design of a cricket microrobot, Proceedings 2000 ICRA. Millennium Conference. IEEE International Conference on Robotics and Automation. Symposia Proceedings (Cat. No.00CH37065) (2000) 110914 vol.2.

[178] T. Chishiro, T. Ono, S. Konishi, leee, PANTOGRAPH MECHANISM FOR CONVERSION FROM SWELLING INTO CONTRACTION MOTION OF PNEUMATIC BALLOON ACTUATOR, 26th IEEE International Conference on Micro Electro Mechanical Systems (MEMS), Taipei, TAIWAN, 2013, pp. 532-535.

[179] K. Takemura, S. Yokota, K. Edamura, A Micro Artificial Muscle Actuator using Electro-conjugate Fluid, Robotics and Automation, 2005. ICRA 2005. Proceedings of the 2005 IEEE International Conference on, 2005, pp. 532-537.

[180] B. Verrelst, R. Van Ham, B. Vanderborght, F. Daerden, D. Lefeber, J. Vermeulen, The pneumatic biped "Lucy" actuated with pleated pneumatic artificial muscles, Autonomous Robots 18(2) (2005) 201-213.

[181] A.A. M. Faudzi, R.F. Surakusumah, D.E. Octorina Dewi, I.N.A. Mohd. Nordin, M.R. Muhammad Razif, Development of Flexible Bronchoscope Device Using Soft Actuator, in: W.K. Lai, E.D. Octorina Dewi (Eds.), Medical Imaging Technology: Reviews and Computational Applications, Springer Singapore, Singapore, 2015, pp. 223-241. 


\section{Tables}

Table 1 Overview of deformation modelling in literature using the finite element method (FEM).

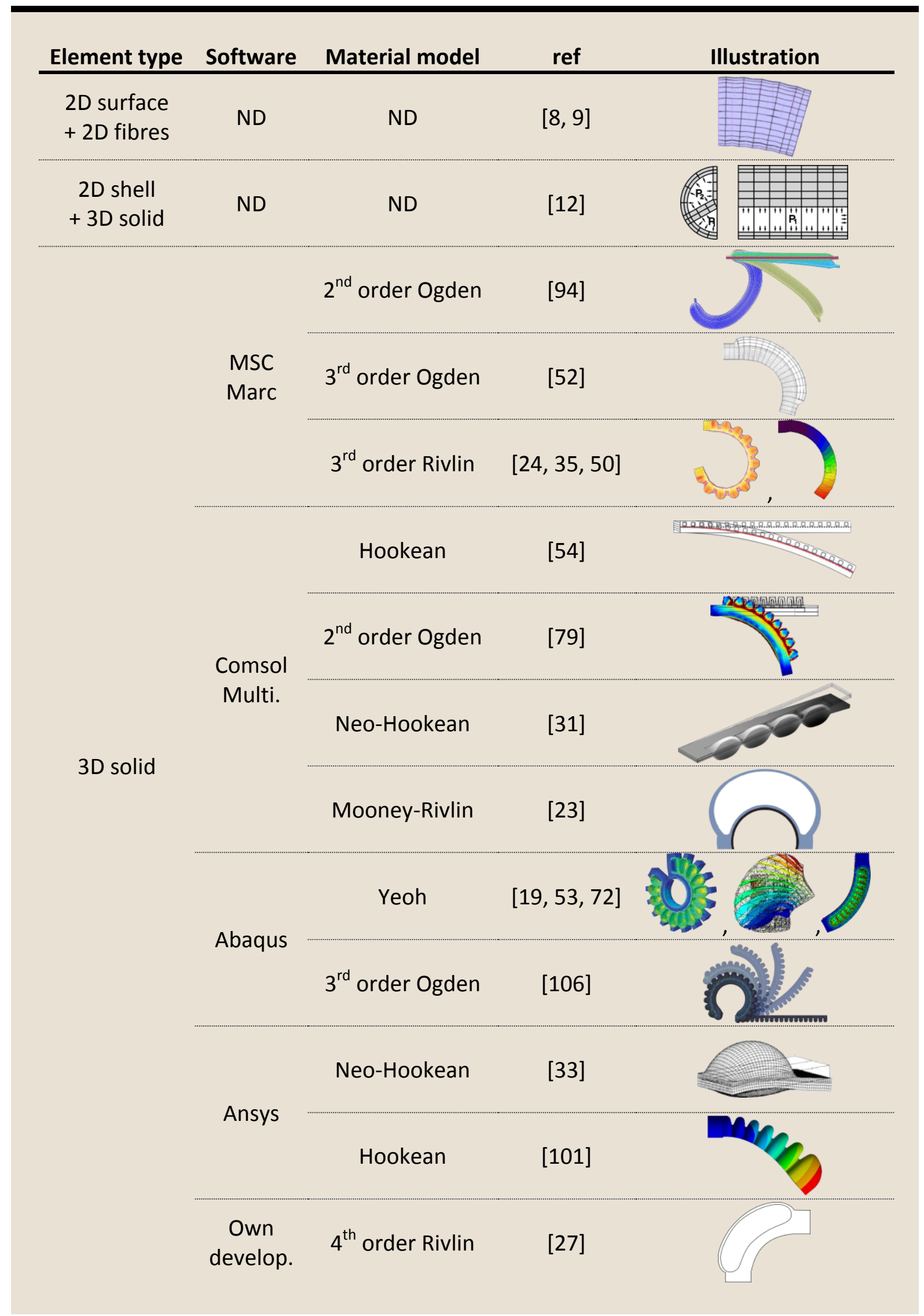




\section{Figures}

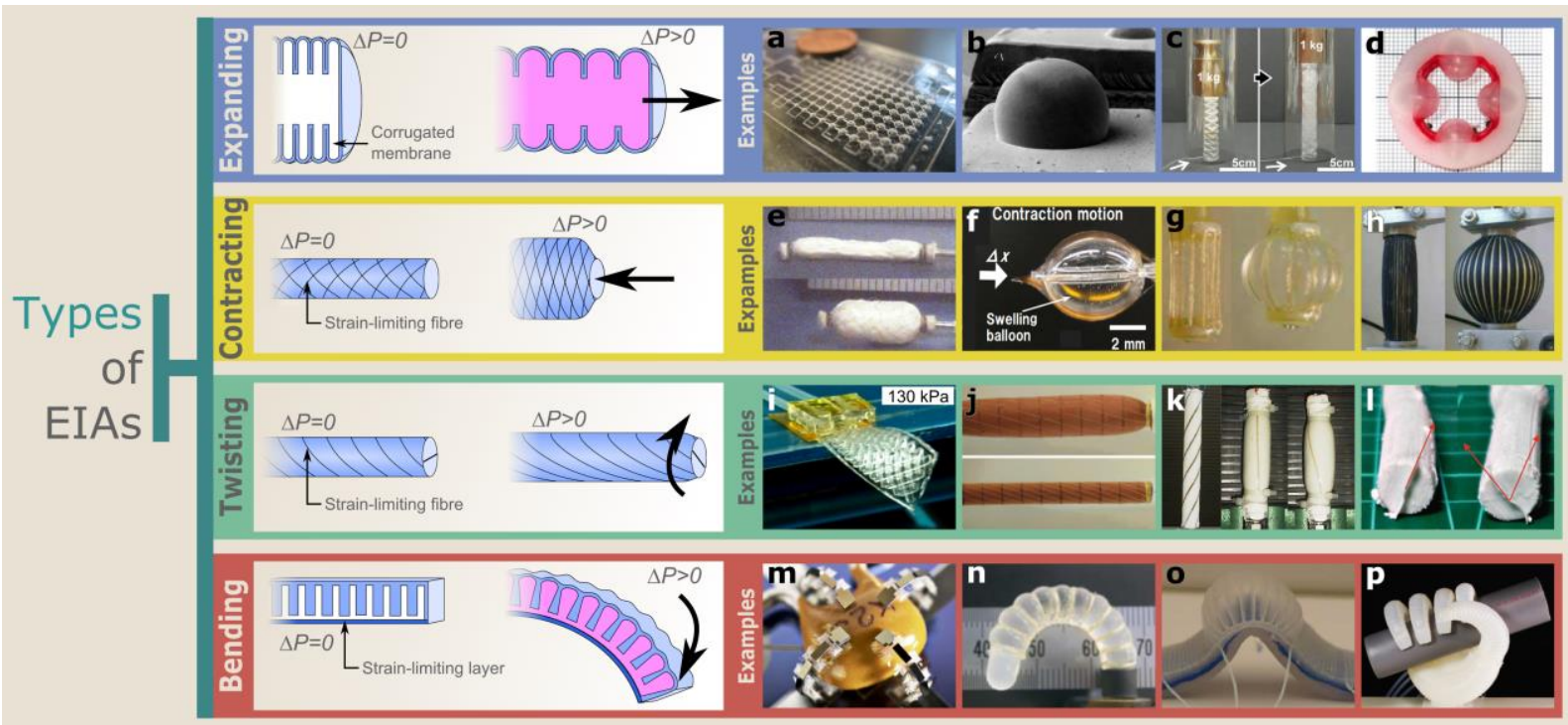

Fig. 1 Classification of elastic inflatable actuators based on their overall deformation. Expanding actuators concentrate their volume increase in one direction. For contracting actuators, a radial expansion is converted by strain-limiting elements into an axial contraction. A spiralling constraint imposes a twisting deformation on the actuator. An asymmetric crosssection converts the volume increase into bending towards the side with the highest bending stiffness. Examples of this classification: a, Array of Braille actuators [175]. b, Osmotic micro-actuator [176]. c, Origami extension actuator [23]. d, Stator for pneumatic rotary actuator [17]. e, McKibben artificial muscle [177]. f, Pantograph actuator [178]. g, Artificial muscle using electro-conjugate fluid [179]. $\mathbf{h}$, Pleated pneumatic artificial muscle [180]. i, Single material twisting actuator [7]. j, Twisting actuator with embedded fibres [13]. k, Actuator with spiralling constraint [9]. I, Flexible bronchoscope device using soft actuator [181]. m, Microhand with internal visual system [141]. $\mathbf{n}$, Robot hand using electro-conjugate fluid [22]. o, Crawling robot [83]. p, Robotic hand for dexterous grasping [149].

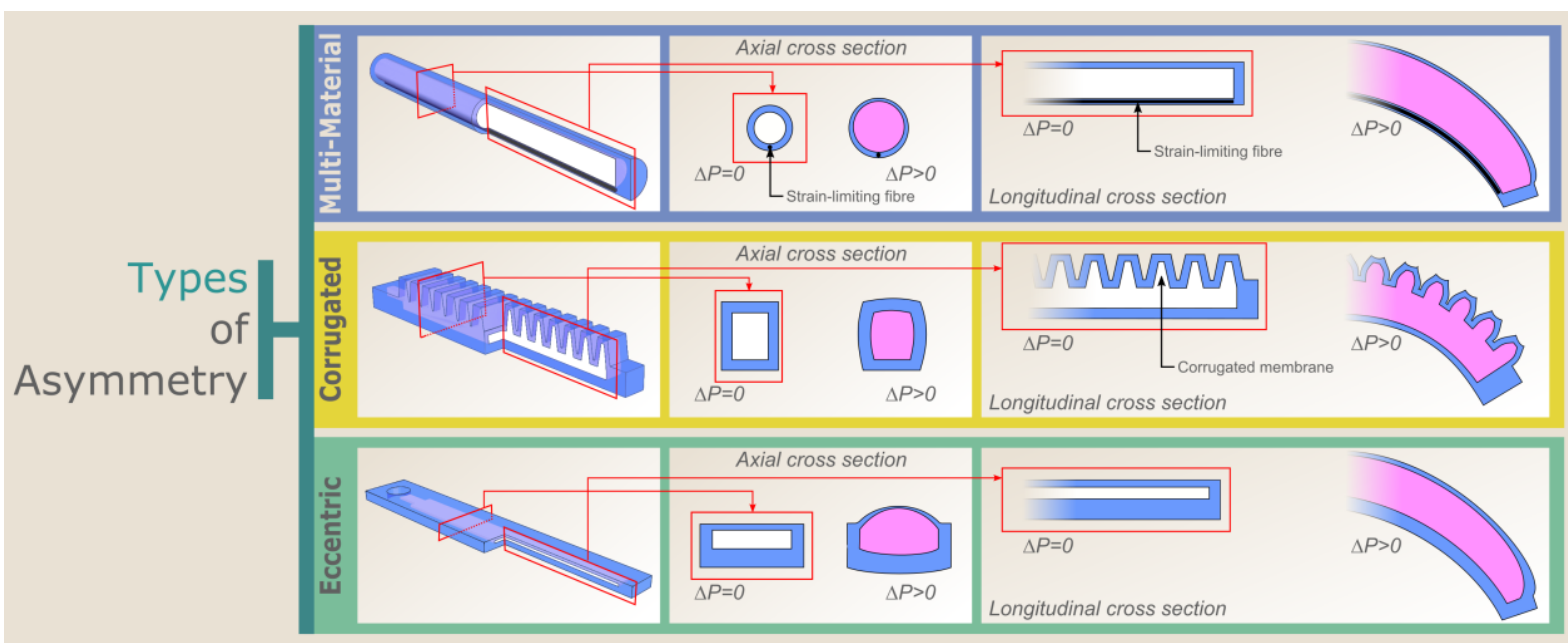

Fig. 2 Different types of asymmetry for elastic inflatable actuators with a bending deformation. Multi-material asymmetry is characterized by combining materials with different Young's moduli that are asymmetrically organized around a central void. Corrugated membrane asymmetry is formed by incorporating geometrical compliant features on one side of the central void. Eccentric void asymmetry is obtained when the pressure chamber is placed eccentrically in the surrounding elastic structure. 


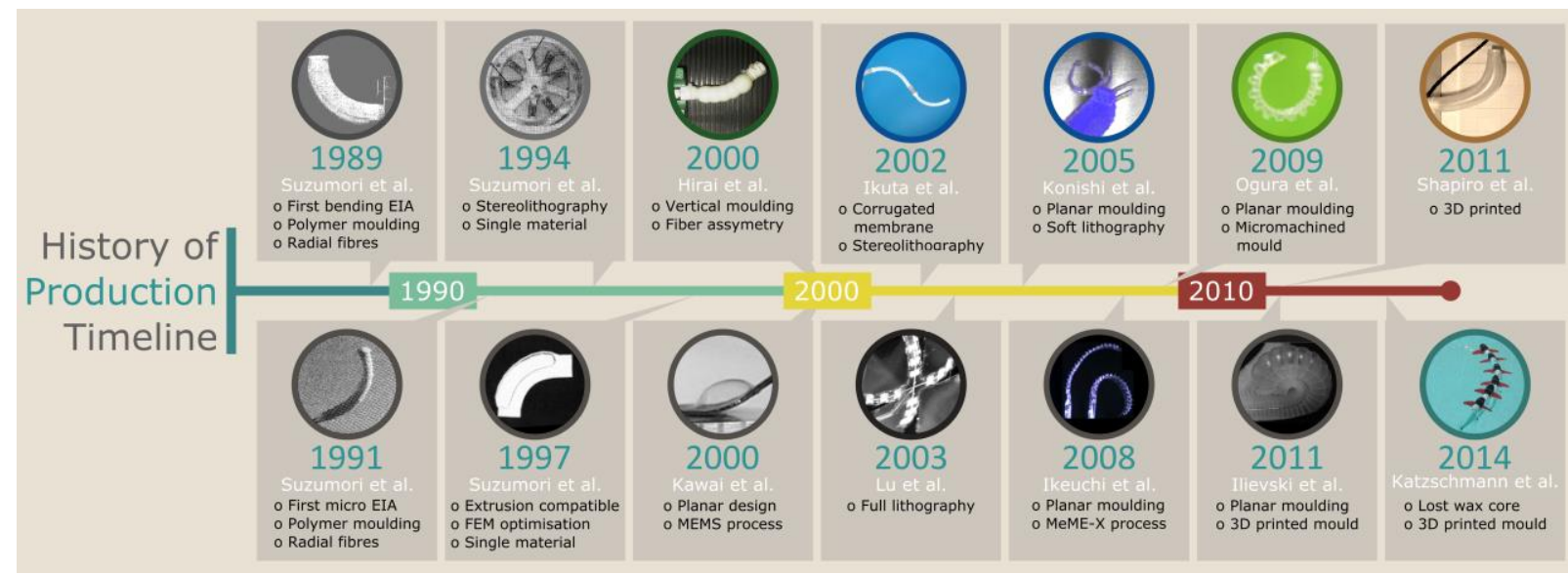

Fig. 3 Timeline showing major production advances in the field of elastic inflatable actuators with a bending deformation. Pictures from: Suzumori (1989) [57], Suzumori (1991) [39], Suzumori (1994) [59], Suzumori (1997) [27], Hirai (2000) [8], Kawai (2000) [61], Ikuta (2002) [60], Lu (2003) [64], Konishi (2005) [62], Ikeuchi (2008) [25], Ogura (2009) [35], Ilievski (2011) [63], Shapiro (2011) [38], , Katzschamnn (2014) [77].
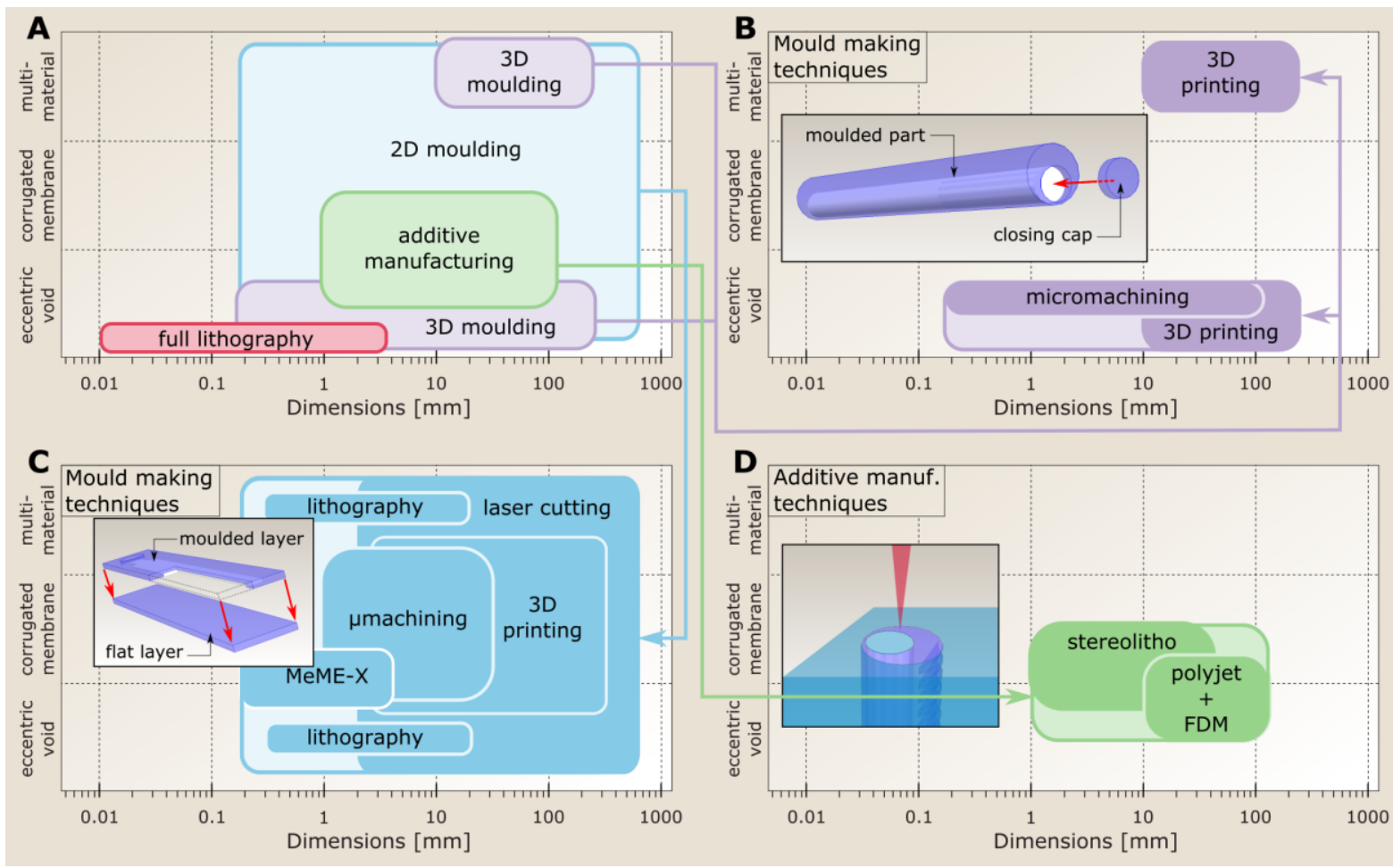

Fig. 4 A. Classification of bending EIA production processes depending on outer dimensions and type of asymmetry, based on bending actuator production literature. Subdivisions of these production process are shown for actuators made by $3 \mathrm{D}$ moulding (B, different mould making techniques), 2D moulding ( $C$, different mould making techniques) and additive manufacturing (D, different printing techniques). 

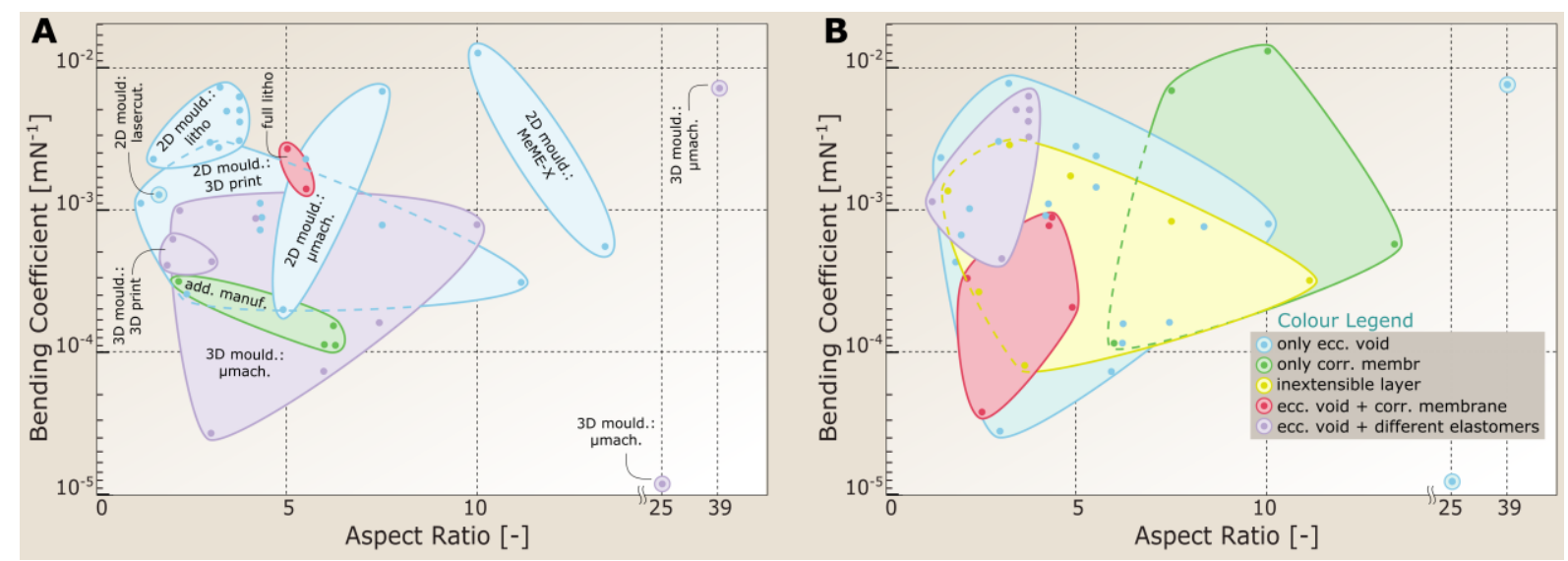

Fig. 5 Bending Coefficient of EIAs A. versus aspect ratio and B. versus asymmetry generation method. Bending coefficients are calculated as the ratio of output curvature over the input pressure at a bending deformation of $90^{\circ}$. Data points are derived from $[14-16,22,24,25,27,28,31,35,36,38,40-44,50,52,57,60,62,65,66,70,72,75,79,88,92,93$, $95-98,100,101,106,127,141,143,147,148,159,162]$. 


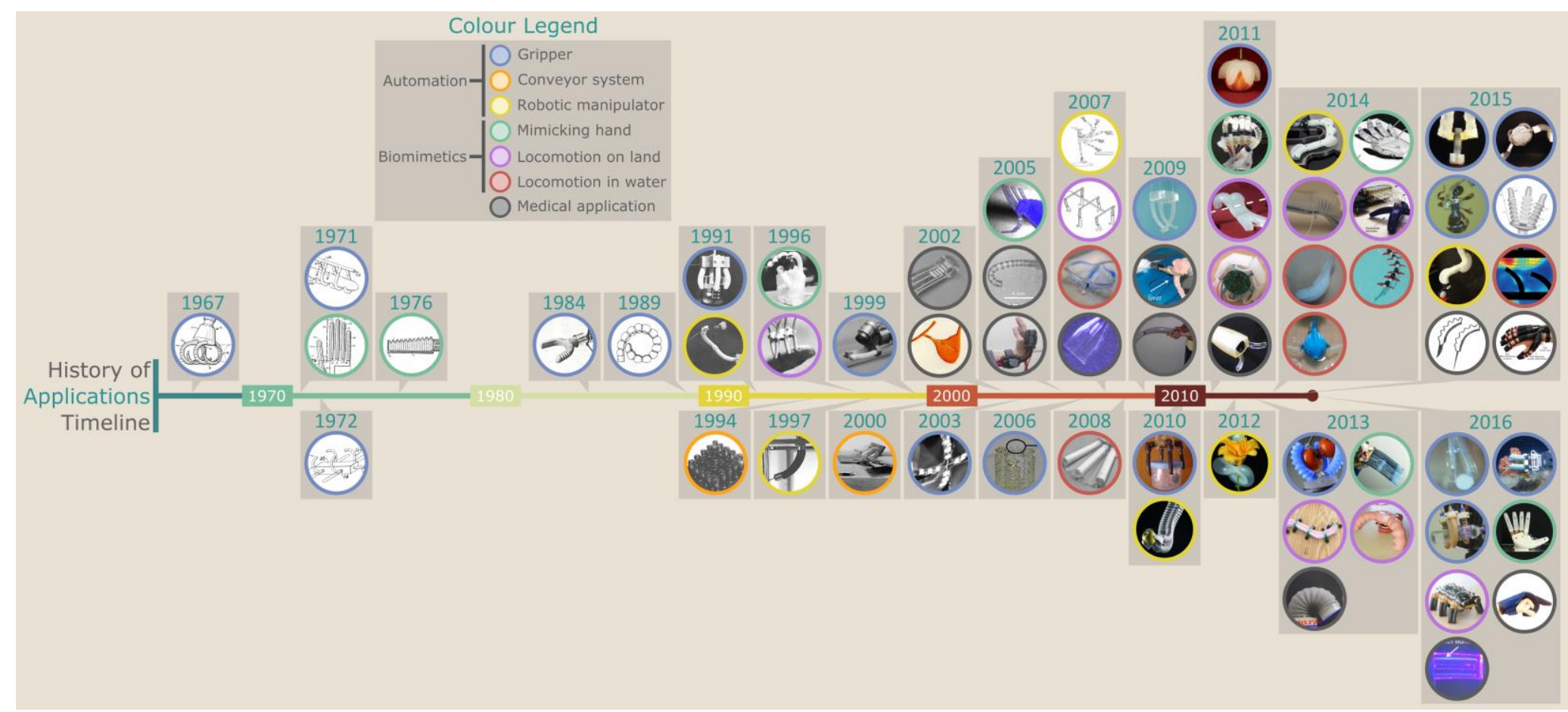

Fig. 6 Timeline showing applications in literature and industry for elastic inflatable actuators with a bending deformation. Application domains are colour coded as shown in the legend. Applications (chronologically, then top to bottom): 1967, material handling apparatus [56]. 1971, Gripping device for grasping delicate objects such as missiles [130]; prosthetic hand [34]. 1972, repeatable gripper [129]. 1976, robot finger [146]. 1984, Bridgestone gripper [132]. 1989, Grappling device for use in outer space [131]. 1991, multi-fingered robotic gripper [39, 133]; robotic manipulator with seven degrees of freedom (DOF) [12]. 1994, 5x5 conveyer system [59]. 1996, human-like hand [45]; miniature walking robot [45]. 1997, 3 DOF spatial positioner [144]. 1999, microgripper for micro inspection robot [134]. 2000, conveyance system [61, 112]. 2002, cuff actuator for adaptive holding around nerves [158]; multi-segment safety-active catheter [25, 60, 96, 127]. 2003, four-fingered microgripper [64, 139-141]. 2005, all PDMS microhand [14, 62, 147, 148]; active bending catheter [68]; active support splint [65] 2006, three-tip microgripper [26]. 2007, robotic arm [40]; six-legged walking machine [40]; manta swimming robot [50]; micro-actuator for retinal pigment epithelium transplantation [90, 160]. 2008, biomimetic pectoral fin actuators [52]. 2009, miniature soft hand [24]; forceps equipped with telescopic motion bending actuators [30]; Colo(noscopy)bot [114]. 2010, four-fingered robotic gripper [70]; bionic handling assistant [145]. 2011, PneuNet gripper [63, 135]; robot hand using electro-conjugate fluid [22, 66]; multigait soft robot [80, 99, 135, 152]; rolling mobile robot with six bending actuators [84]; retractor for spacing in front of endoscope or for submucosal dissection [161]. 2012 Robotic tentacle [73]. 2013, commercial soft robotic gripper by Soft Robotic Inc.; highly compliant robotic hand [88]; soft snake robot [155]; soft explosive jumper [21, 81]; STIFF-FLOP surgical manipulator [36, 72]. 2014, 2D whole arm soft robotic manipulator [74]; planar printable robotic hand [107]; crawling locomotion [83]; combining hard and soft robots [154]; caudal fin [53]; soft robotic fish [76, 77]; fish robot [95]. 2015, soft microgripper [49]; robotic gripper consisting of eight bending actuators [79]; microrobotic tentacles [43]; 3D printed gripping apparatus by Materialise NV [105]; 3D whole arm soft robotic manipulator [75]; artificial cilia array for biomimetic fluid propulsion [20]; soft robotic medical needle or knife [136]; soft robotic glove for rehabilitation [16]. 2016, cell manipulation system [142]; soft robotic grippers for sampling on deep reefs [137]; variable stiffness robotic gripper [138]; underactuated robotic dexterous hand [149]; dexterous soft robotic leg [156]; Exoskeleton for rehabilitation [106]; flexible scanner for endoscopic imaging [162]. 